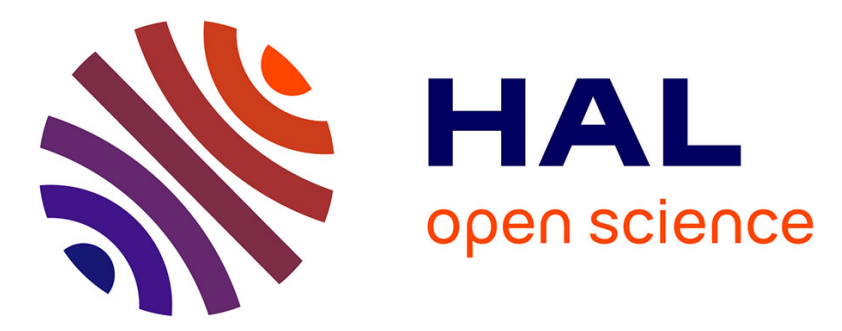

\title{
Transmission and tunneling probability in two-band metals: Influence of magnetic breakdown on the Onsager phase of quantum oscillations
}

Jean-Yves Fortin, Alain Audouard

\section{- To cite this version:}

Jean-Yves Fortin, Alain Audouard. Transmission and tunneling probability in two-band metals: Influence of magnetic breakdown on the Onsager phase of quantum oscillations. Low Temperature Physics, 2017, 43 (2), pp.173-185. 10.1063/1.4976631 . hal-02264901

\section{HAL Id: hal-02264901 \\ https://hal.science/hal-02264901}

Submitted on 28 Aug 2019

HAL is a multi-disciplinary open access archive for the deposit and dissemination of scientific research documents, whether they are published or not. The documents may come from teaching and research institutions in France or abroad, or from public or private research centers.
L'archive ouverte pluridisciplinaire HAL, est destinée au dépôt et à la diffusion de documents scientifiques de niveau recherche, publiés ou non, émanant des établissements d'enseignement et de recherche français ou étrangers, des laboratoires publics ou privés. 
 \\ Transmission and tunneling probability in two-band metals: Influence of magnetic breakdown on the Onsager phase of quantum oscillations}

Cite as: Low Temp. Phys. 43, 173 (2017); https://doi.org/10.1063/1.4976631

Submitted: 19 July 2016 . Accepted: 26 January 2017 . Published Online: 15 March 2017

Jean-Yves Fortin, and Alain Audouard

ARTICLES YOU MAY BE INTERESTED IN

Shubnikov-de Haas oscillations and electronic correlations in the layered organic metal $\mathrm{K}^{-}$ $(\mathrm{BETS})_{2} \mathrm{Mn}\left[\mathrm{N}(\mathrm{CN})_{2}\right]_{3}$

Low Temperature Physics 43, 239 (2017); https://doi.org/10.1063/1.4976634

Spin resonance and spin fluctuations in a quantum wire

Low Temperature Physics 43, 211 (2017); https://doi.org/10.1063/1.4976632

High-frequency resonances and weakly damped collective modes in highly anisotropic Q1D conductors

Low Temperature Physics 43, 186 (2017); https://doi.org/10.1063/1.4977209

LOW TEMPERATURE TECHNIQUES OPTाCAL CAVITY PHVSICS MITIGATING THERMAL \& VIBRATIONAL NOISE

DOWNLOAD THE WHITE PAPER 


\title{
Transmission and tunneling probability in two-band metals: Influence of magnetic breakdown on the Onsager phase of quantum oscillations
}

\author{
Jean-Yves Fortin ${ }^{\text {a) }}$ \\ Institut Jean Lamour, Département de Physique de la Matière et des Matériaux, Groupe de Physique \\ Statistique, CNRS-Nancy-Université BP 70239, F-54506 Vandoeuvre les Nancy Cedex, France
}

Alain Audouard ${ }^{\text {b) }}$

Laboratoire National des Champs Magnétiques Intenses (UPR 3228 CNRS, INSA, UGA, UPS), 143 Ave. de Rangueil, F-31400 Toulouse, France

(Submitted July 19, 2016)

Fiz. Nizk. Temp. 43, 211-226 (February 2017)

\begin{abstract}
Tunneling amplitude through magnetic breakdown (MB) gap is considered for two-bands Fermi surfaces illustrated in many organic metals. In particular, the S-matrix associated to the wave function transmission through the MB gap for the relevant class of differential equations is the main object allowing the determination of tunneling probabilities and phases. The calculated transmission coefficients include a field-dependent Onsager phase. As a result, quantum oscillations are not periodic in $1 / B$ for finite magnetic breakdown gap. Exact and approximate methods are proposed for computing ratio amplitudes of the wave function in interacting two-band models. Published by AIP Publishing. [http://dx.doi.org/10.1063/1.4976631]
\end{abstract}

\section{INTRODUCTION}

In recent years, interest regarding determination of the quantum oscillations phase has been renewed. This was in particular motivated by the observation of a Berry phase both in three-dimensional metals ${ }^{1}$ and topological insulators, ${ }^{2}$ for example in the case of Dirac fermions. ${ }^{3}$ One might add the effect of non-parabolicity of the dispersion equation which, both in conventional fermions and, especially, in Dirac fermions is liable to induce phase offsets. ${ }^{4}$

The problem of the Onsager phase was nevertheless addressed much earlier, regarding the effect of the phase offset induced by magnetic breakdown (MB). ${ }^{5-7}$ The case of the model Fermi surface (FS), known as the linear chain of coupled orbits by Pippard, ${ }^{8}$ is addressed in Refs. 5 and 6 . As it is well known, the first experimental realization of this FS topology was observed in the organic conductor $\kappa-(\mathrm{ET})_{2} \mathrm{Cu}(\mathrm{SCN})_{2}$, where ET stands for the bis-ethylenedithio-tetrathiafulvalene molecule. ${ }^{9}$ In addition to the $\pi / 2$ dephasing occurring at each $\mathrm{MB}$ reflection, it was demonstrated that a field-dependent phase offset should be observed ${ }^{5}$ as it has been checked for $\theta-(\mathrm{ET})_{4} \mathrm{CoBr}_{4}\left(\mathrm{C}_{6} \mathrm{H}_{4} \mathrm{Cl}_{2}\right) .{ }^{10}$

The main objective of this article is to consider the tunneling phenomena in interacting cyclotronic orbits, and its implication to the wave-function characteristics at high and low field limits. In the first step of this paper, we review the problem of transmission and reflection coefficients within the S-matrix theory, when a particle coming from infinity is scattered by a tunneling region. From the simple model due to Rosen-Zener ${ }^{12}$ and applied later to the magnetic breakdown case, ${ }^{5,13}$ we focus on the effect of phase divergence in the S-matrix amplitudes. This actually occurs in different fields of physics, for example the level-crossing problem. ${ }^{14}$ Amplitude ratio of the wave function is then considered in the second step when multiple paths are involved in the tunneling process, leading to an oscillatory behavior of the transmission coefficient. High field and semiclassical results are presented and compared to the numerical resolution of the Schrodinger equation. In the third step, we consider an exact approach to compute the quantum states in the interacting case of two circular orbits with bound state conditions. This new method is based on an extension of the usual (creation and annihilation) bosonic operators of the harmonic oscillator that includes effective coupling between the individual Fermi surfaces using two parameters, representing the coupling itself and the gap separately. This is an approach that can be easily generalized to a linear chain of coupled orbits, and which should give new insights on the wave-function properties. Finally, consequences on experimental de Haas-van Alphen oscillations phase offset are considered for real FS of organic conductors.

\section{REVIEW OF THE TRANSMISSION PHENOMENA IN A SIMPLE TWO-BAND MODEL}

The presented model is intended to review the local transmission phenomena in two-band metals with MB junctions, the FS of which achieves a linear chain of coupled orbits (see, e.g., Refs. 9, 15, and 16). A typical example of such Fermi surface is presented in Fig. 1 for (BEDO-TTF $)_{5}$ $\left[\mathrm{CsHg}(\mathrm{SCN})_{4}\right]_{2}$ (Ref. 11) (BEDO-TTF stands for the bis-ethylenedioxi-tetrathiafulvalene molecule), where an incoming amplitude (a) is transmitted to (b) and reflected to (c). At the vicinity of the MB junction, two linear sheets hybridized with energy constant $\varepsilon_{g}$ can be considered. The local Fermi surface is represented on Fig. 2 for a non-zero coupling, and the linearized effective Hamiltonian can be written as

$$
\hat{H}\left(\begin{array}{c}
\varphi_{1} \\
\varphi_{2}
\end{array}\right)=\left(\begin{array}{cc}
k_{y}+k_{x} & \varepsilon_{g} \\
\varepsilon_{g} & k_{y}-k_{x}
\end{array}\right)\left(\begin{array}{l}
\varphi_{1} \\
\varphi_{2}
\end{array}\right)=\left(\begin{array}{l}
0 \\
0
\end{array}\right) .
$$

For $\varepsilon_{g}=0$, the two sheets and the wave functions $\varphi_{1}$ and $\varphi_{2}$ are independent. In such case, the MB gap which is proportional to $\varepsilon_{g}^{2}$, is zero. In presence of a magnetic field, the 


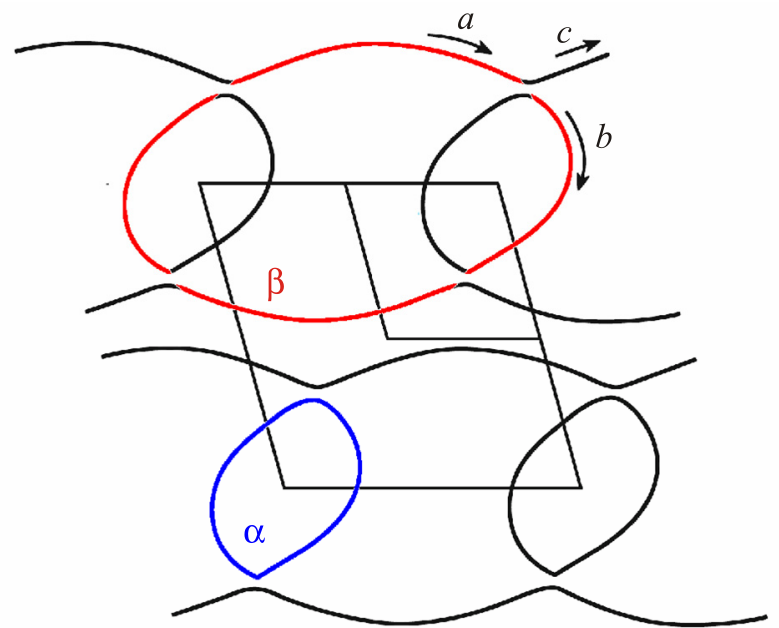

FIG. 1. Fermi surface of the organic conductor (BEDO-TTF $)_{5}\left[\mathrm{CsHg}(\mathrm{SCN})_{4}\right]_{2}$ (from Ref. 11). An incoming wave (a) on the P orbit is reflected in (c) and transmitted to the a orbit (b).

quantum representation of this model is chosen such that $y=$ $k_{y}$ and $\hat{x}=\hat{k}_{x}=2 i \pi b \partial_{y}$, with $b=e B /(2 \pi \hbar)$. In this case, the differential equations for the wave functions are

$$
\begin{gathered}
\left(y+i h \frac{\partial}{\partial y}\right) \varphi_{1}+\varepsilon_{g} \varphi_{2}=0 \quad \text { and } \\
\varepsilon_{g} \varphi_{1}+\left(y-i h \frac{\partial}{\partial y}\right) \varphi_{2}=0,
\end{gathered}
$$

where $h=2 \pi b$ is an effective magnetic Planck constant. This set of first-order differential equations can be reduced using the transformation $\varphi_{1}=\mathrm{e}^{i y^{2} / 2 h} g_{1}(y)$ and $\varphi_{2}=\mathrm{e}^{-i y^{2} / 2 h}$ $g_{2}(y)$, where now

$$
\left(\begin{array}{l}
g_{1}^{\prime} \\
g^{\prime}{ }_{2}
\end{array}\right)=\frac{\varepsilon_{g}}{h}\left(\begin{array}{cc}
0 & i \mathrm{e}^{-i y^{2} / h} \\
-i \mathrm{e}^{i y^{2 / h}} & 0
\end{array}\right)\left(\begin{array}{l}
g_{1} \\
g_{2}
\end{array}\right)=\frac{\varepsilon_{g}}{h} U(y)\left(\begin{array}{l}
g_{1} \\
g_{2}
\end{array}\right),
$$

where $U$ is a unitary matrix. We can notice that the product $U\left(y_{1}\right) U\left(y_{2}\right)$ is diagonal, which makes easier the computation of any multiple products of $U(y)$

$$
U\left(y_{1}\right) U\left(y_{2}\right)=\left(\begin{array}{cc}
\mathrm{e}^{-i y_{1}^{2} / h+i y_{2}^{2} / h} & 0 \\
0 & \mathrm{e}^{i y_{1}^{2} / h-i y_{2}^{2} / h}
\end{array}\right) .
$$

The solution of Eq. (3) is given by a series of matrix ordered products and multiple integrals ${ }^{17}$

$$
\begin{aligned}
& \left(\begin{array}{l}
g_{1}(y) \\
g_{2}(y)
\end{array}\right)=\left(1+\frac{\varepsilon_{g}}{h} \int_{-y}^{y} d y_{1} U\left(y_{1}\right)+\frac{\varepsilon_{g}^{2}}{h^{2}} \int_{-y}^{y} d y_{1}\right. \\
& \left.\times \int_{-y}^{y_{1}} d y_{2} U\left(y_{1}\right) U\left(y_{2}\right)+\cdots\right)\left(\begin{array}{l}
g_{1}(-y) \\
g_{2}(-y)
\end{array}\right) \text {. }
\end{aligned}
$$

* $h$ is not to be confounded with the real Planck constant that we will write $2 \pi \hbar$ in the rest of the paper.

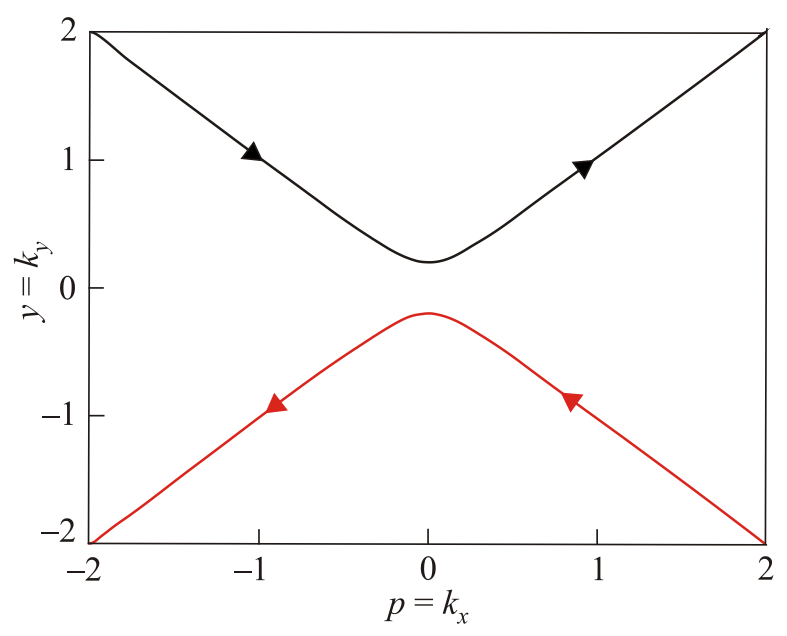

FIG. 2. Effective two-band model. The hybridization parameter is $\varepsilon_{g}=0.2$. The arrows represent the increase or decrease of the phase, specifically the gradient of $\pm y^{2} / 2 h$. Here are represented two electronic bands with trigonometric orientation of the trajectories.

Using the property Eq. (4) and setting $\omega(y)=y^{2}$ ( $\omega$ can be a more general function of $y$ as we shall see later), one can write a transfer or S-matrix between two points $-y$ and $y>$ 0 on the axis, away from the tunneling region

$$
\left(\begin{array}{l}
g_{1}(y) \\
g_{2}(y)
\end{array}\right)=\left(\begin{array}{ll}
t & s \\
\bar{s} & \bar{t}
\end{array}\right)\left(\begin{array}{l}
g_{1}(-y) \\
g_{2}(-y)
\end{array}\right)
$$

with $t \bar{t}-s \bar{s}=1$ by conservation of probabilities. The matrix elements are infinite sums of ordered integrals given by

$$
\begin{aligned}
t= & 1+\frac{\varepsilon_{g}^{2}}{h^{2}} \int_{-y}^{y} d y_{1} \int_{-y}^{y_{1}} d y_{1} \mathrm{e}^{-i \omega\left(y_{1}\right) / h+\mathrm{i} \omega\left(y_{2}\right) / h}+\cdots, \\
s= & \frac{\varepsilon_{g}}{h} \int_{-y}^{y} d y_{1} \mathrm{e}^{-i \omega\left(y_{1}\right) / h}+\frac{\varepsilon_{g}^{3}}{h^{3}} \int_{-y}^{y} d y_{1} \int_{-y}^{y_{1}} d y_{2} \int_{-y}^{y_{2}} d y_{3} \\
& \times \mathrm{e}^{-i \omega\left(y_{1}\right) / h+i \omega\left(y_{2}\right) / h-i \omega\left(y_{3}\right) / h}+\cdots,
\end{aligned}
$$

where the $y_{i}$ are dummy variables. The characteristics of this matrix have been studied by many authors ${ }^{18,19}$ in the case of the Zener effect. ${ }^{12}$ In the Gaussian case, when $\omega(y)$ is quadratic, it is convenient to use the theta function representation in the complex plane ${ }^{18}$ when $y=\infty$. Indeed the diagonal matrix element $t=\bar{t}$ can then be computed with the aid of simple translation transformations. For example, the double integral in the first line of Eq. (7) can be simplified by introducing $\theta(x)=\oint \frac{d Z}{2 i \pi(Z-i \varepsilon)} \mathrm{e}^{i z x}$, where the path in located on the upper half complex plane, to satisfy the constraint $y_{1}>y_{2}$

$$
\begin{aligned}
& \int_{-\infty}^{\infty} d y_{1} \int_{-\infty}^{y_{1}} d y_{2} \mathrm{e}^{-i \omega\left(y_{1}\right) / h+i \omega\left(y_{2}\right) / h} \\
& =\int_{-\infty}^{\infty} d y_{1} \int_{-\infty}^{\infty} d y_{2} \oint \frac{d z}{2 i \pi} \frac{\mathrm{e}^{-i \omega\left(y_{1}\right) / h+i \omega\left(y_{2}\right) / h+i\left(y_{1}-y_{2}\right) z}}{z-i \varepsilon} \\
& =(\pi h) \oint \frac{d z}{2 i \pi} \frac{e^{i h z^{2} / 4}}{z-i \varepsilon}=\frac{\pi h}{2} .
\end{aligned}
$$


The last integral is obtained after translating $y_{1} \rightarrow y_{1}+h z / 2$ and $y_{2} \rightarrow y_{2}-h z / 2$, respectively, to remove the couplings with $z$. Then $t=1+\frac{\pi \varepsilon_{g}^{2}}{2 h}+\cdots$. All the terms in the series can be computed similarly, and the resummation leads to $t=$ $\mathrm{e}^{\pi \varepsilon_{g}^{2} / 2 h}$. We will introduce in the following the breakdown field $h_{0}=\pi \varepsilon_{g}^{2}$ which is characteristic of the tunneling process. The same techniques could be applied for elements $s$, but one finds that the result is diverging in the large $y$ limit. The reason is that the phase of $\mathrm{s}$ is diverging logarithmically, ${ }^{14}$ as we will see below, although the modulus is finite. A correct asymptotic analysis for finite $y$ and $-y$ is therefore needed.

\section{A. Asymptotic analysis}

One can solve the equation for $g_{1}$ and $g_{2}$ using standard techniques. Indeed, the differential equation satisfied by $g_{1}$ can be obtained, separating $g_{1}$ from $g_{2}$ in Eq. (3)

$$
g_{1}^{\prime \prime}+\frac{2 i y}{h} g_{1}^{\prime}=\frac{\varepsilon_{g}^{2}}{h^{2}} g_{1}, \quad g_{2}=\frac{h}{i \varepsilon_{g}} \mathrm{e}^{i y^{2} / h} g_{1}^{\prime}
$$

The two odd and even solutions for $g_{1}$ are a combination of two Kummer functions $M^{20}$ with an imaginary variable, and which can be chosen such that

$$
g_{1}(y)=A M\left(\frac{i \varepsilon_{g}^{2}}{4 h}, \frac{1}{2}, \frac{i y^{2}}{h}\right)+B y M\left(\frac{1}{2}+\frac{i \varepsilon_{g}^{2}}{4 h}, \frac{3}{2},-\frac{i y^{2}}{h}\right),
$$

where $A$ and $B$ are constants. Then $\varphi_{1}=\mathrm{e}^{i y^{2} / 2 h} g_{1}$ and $\varphi_{2}=\mathrm{e}^{i y^{2} / 2 h} g_{2}$. We notice that there are only two constants in the problem, since from Eq. (9) $g_{2}$ is entirely determined by $g_{1}$. The S-matrix (6) between points $-y$ and $y>0$ can then be obtained by eliminating the coefficients $A$ and $B$ in Eq. (10). Setting

$$
g_{1}( \pm y)=A a_{1} \pm B b_{1}, \quad g_{2}( \pm y)= \pm A a_{2}+B b_{2}
$$

one can express the outgoing wave function $g_{1}(-y)$ and $g_{2}(y)$ as function of an incoming wave function $g_{1}(y)$ and $g_{2}(-y)$ as represented locally in Fig. 1

$$
\left(\begin{array}{c}
g_{1}(-y) \\
g_{2}(y)
\end{array}\right)=\left(\begin{array}{cc}
1 / t & -s / t \\
\bar{s} / t & 1 / t
\end{array}\right)\left(\begin{array}{c}
g_{1}(y) \\
g_{2}(-y)
\end{array}\right)=M\left(\begin{array}{c}
g_{1}(y) \\
g_{2}(-y)
\end{array}\right) .
$$

The functions $\left(a_{1}, a_{2}, b_{1}, b_{2}\right)$ depending on $y$ are given by Kummer functions

$$
\begin{aligned}
a_{1}= & M\left(\frac{i \varepsilon_{g}^{2}}{4 h}, \frac{1}{2},-\frac{i y^{2}}{h}\right), \quad b_{1}=y M\left(\frac{1}{2}+\frac{i \varepsilon_{g}^{2}}{4 h}, \frac{3}{2},-\frac{i y^{2}}{h}\right), \\
a_{2}= & -\frac{2 y^{2}}{3 \varepsilon_{g}} e^{i y^{2} / h}\left(1+\frac{i \varepsilon_{g}^{2}}{2 h}\right) M\left(\frac{3}{2}+\frac{i \varepsilon_{g}^{2}}{4 h}, \frac{5}{2},-\frac{i y^{2}}{h}\right) \\
& +\frac{h}{i \varepsilon_{g}} e^{i y^{2} / h} M\left(\frac{1}{2}+\frac{i \varepsilon_{g}^{2}}{4 h}, \frac{3}{2},-\frac{i y^{2}}{h}\right) \\
b_{2}= & -y \frac{i \varepsilon_{g}}{h} e^{i y^{2} / h} M\left(1+\frac{i \varepsilon_{g}^{2}}{4 h}, \frac{3}{2},-\frac{i y^{2}}{h}\right),
\end{aligned}
$$

and the expression for the S-matrix elements is given by

$$
\begin{gathered}
t=\bar{t}=\frac{a_{1} a_{2}+b_{1} b_{2}}{a_{1} a_{2}-b_{1} b_{2}}, \quad s=\frac{2 a_{1} b_{1}}{a_{1} a_{2}-b_{1} b_{2}}, \\
\bar{s}=\frac{2 a_{2} b_{2}}{a_{1} a_{2}-b_{1} b_{2}}, \quad t^{2}-s \bar{s}=1 .
\end{gathered}
$$

Asymptotically, for $y$ large, one can use the expansion ${ }^{21}$

$$
M(a, b, z) \simeq \frac{\Gamma(b)}{\Gamma(b-a)}(-z)^{-a}+\frac{\Gamma(b)}{\Gamma(a)} e^{z} z^{a-b}
$$

and keep the dominant terms

$$
\begin{aligned}
g_{1}( \pm y) \simeq & \sqrt{\pi}\left(\frac{i y^{2}}{h}\right)^{-i \varepsilon_{g}^{2} / 4 h} \\
& \times\left(\frac{A}{\Gamma\left(\frac{1}{2}-i \varepsilon_{g}^{2} / 4 h\right)} \pm \frac{\sqrt{h}}{2 \sqrt{i}} \frac{B}{\Gamma\left(1-i \varepsilon_{g}^{2} / 4 h\right)}\right)
\end{aligned}
$$

and

$$
\begin{aligned}
& g_{2}( \pm y) \simeq \sqrt{\pi}\left(\frac{-i y^{2}}{h}\right)^{i \varepsilon_{g}^{2} / 4 h} \times\left( \pm \frac{\varepsilon_{g}}{2 \sqrt{i h}} \frac{A}{\Gamma\left(1+i \varepsilon_{g}^{2} / 4 h\right)}\right. \\
& \left.-\frac{i h}{\varepsilon_{g}} \frac{B}{\Gamma\left(\frac{1}{2}+i \varepsilon_{g}^{2} / 4 h\right)}\right) \text {. }
\end{aligned}
$$

Using the different duplication formulas for gamma's functions: $\quad \Gamma((1 / 2)+i x) \Gamma((1 / 2)-i x)=\pi / \cosh (\pi x), \quad \Gamma(i x)$ $\Gamma(1-i x)=\pi / i \sinh (\pi x)$, and $\Gamma((1 / 2)+i x) \Gamma(i x)=\sqrt{\pi} 2^{1-2 i x}$ $\Gamma(2 i x)$, one obtains the probability of tunneling $p=1 /$ $t=\mathrm{e}^{-\pi \varepsilon_{g}^{2} / 2 h}=\mathrm{e}^{-h_{0} / 2 h}$, which is the typical tunneling amplitude already obtained in many previous works. ${ }^{5,13}$ The breakdown field is in this case equal to $h_{0}=\pi \varepsilon_{g}^{2}$ and corresponds exactly to the semiclassical expression (see text further below). The remaining elements of the tunneling matrix $M$ can be obtained after some algebra and one finds the unitary matrix

$$
M=\left(\begin{array}{cc}
p & -i q e^{-i \phi} \\
-i q e^{i \phi} & p
\end{array}\right)
$$

where $q=\sqrt{1-p^{2}}$ and the phase $\phi$ depends on the coordinate $y$

$$
\phi(y)=-\frac{\pi}{4}+\frac{\varepsilon_{g}^{2}}{2 h} \log \left(\frac{2 y^{2}}{h}\right)-\arg \Gamma\left(i \varepsilon_{g}^{2} / 2 h\right) .
$$

The phase diverges logarithmically with $y$. Since the FS is not accounted for by Fig. 2 for $\left|k_{x}\right| \gg 1$ where it should be more curved, we assume that the phase is finite far from the tunneling region. Using a Stirling expansion of the gamma function in Eq. (16), one finds that $\phi$ is finite asymptotically only when $y^{2}=h_{0} \mathrm{e}^{-1} / 4 \pi$. This corresponds approximately to the coordinate where the tunneling region ends, e.g., $y \simeq \varepsilon_{g}$. In this case, instead of Eq. (16), the phase is given by the following regularization: $:^{5,22}$

$$
\phi=-\frac{\pi}{4}+u \log u-u-\arg \Gamma(i u), \quad u=\frac{h_{0}}{2 \pi h} .
$$


The phase is zero in the low field limit ( $u$ large) and equal to $\pi / 4$ when $h$ is large ( $u$ small).

\section{TRANSMISSION THROUGH THE SMALL POCKET}

A more general model is given by an hybridization of two parabolic bands, whose Fermi surface is composed of two circular sheets, each of radius $k_{0}$ and centers $\pm k_{c}$, as displayed in Fig. 3, and for which the Hamiltonian reads

$$
\hat{H}=\left(\begin{array}{cc}
\frac{1}{2}\left(k_{x}+k_{c}\right)^{2}+\frac{1}{2}\left(k_{y}^{2}-k_{0}^{2}\right) & \varepsilon_{g} \\
\varepsilon_{g} & \frac{1}{2}\left(k_{x}-k_{c}\right)^{2}+\frac{1}{2}\left(k_{y}^{2}-k_{0}^{2}\right)
\end{array}\right) .
$$

Rescaling the variables with $k_{c}$ and setting $x=k_{x} / k_{c}$, $y=k_{y} / k_{c}, \varepsilon_{g} / k_{c}^{2} \rightarrow \varepsilon_{g}$, and $y_{0}^{2}=k_{0}^{2} / k_{c}^{2}-1>0$, one obtains

$$
\hat{H}=\left(\begin{array}{cc}
\frac{1}{2}(x+1)^{2}+\frac{1}{2}\left(y^{2}-y_{0}^{2}-1\right) & \varepsilon_{g} \\
\varepsilon_{g} & \frac{1}{2}(x-1)^{2}+\frac{1}{2}\left(y^{2}-y_{0}^{2}-1\right)
\end{array}\right) .
$$

For small $x$, one has the approximation near the tunneling points (points $a, b, a^{\prime}$, and $b^{\prime}$ in Fig. 3)

$$
\hat{H} \simeq\left(\begin{array}{cc}
x+\frac{1}{2}\left(y^{2}-y_{0}^{2}\right) & \varepsilon_{g} \\
\varepsilon_{g} & -x+\frac{1}{2}\left(y^{2}-y_{0}^{2}\right)
\end{array}\right) .
$$

This Hamiltonian gives a first order differential matrix equation, similar to Eq. (3), after setting $\varphi_{1}(y)=\mathrm{e}^{i \omega(y) / 2 h} g_{1}(y)$ and $\varphi_{2}(y)=\mathrm{e}^{-i \omega(y) / 2 h} g_{2}(y)$

$$
\left(\begin{array}{c}
g_{1}^{\prime} \\
g_{2}^{\prime}
\end{array}\right)=\frac{\varepsilon_{g}}{h}\left(\begin{array}{cc}
0 & i \mathrm{e}^{-i \omega(y) / h} \\
-i \mathrm{e}^{i \omega(y) / h} & 0
\end{array}\right)\left(\begin{array}{l}
g_{1} \\
g_{2}
\end{array}\right),
$$

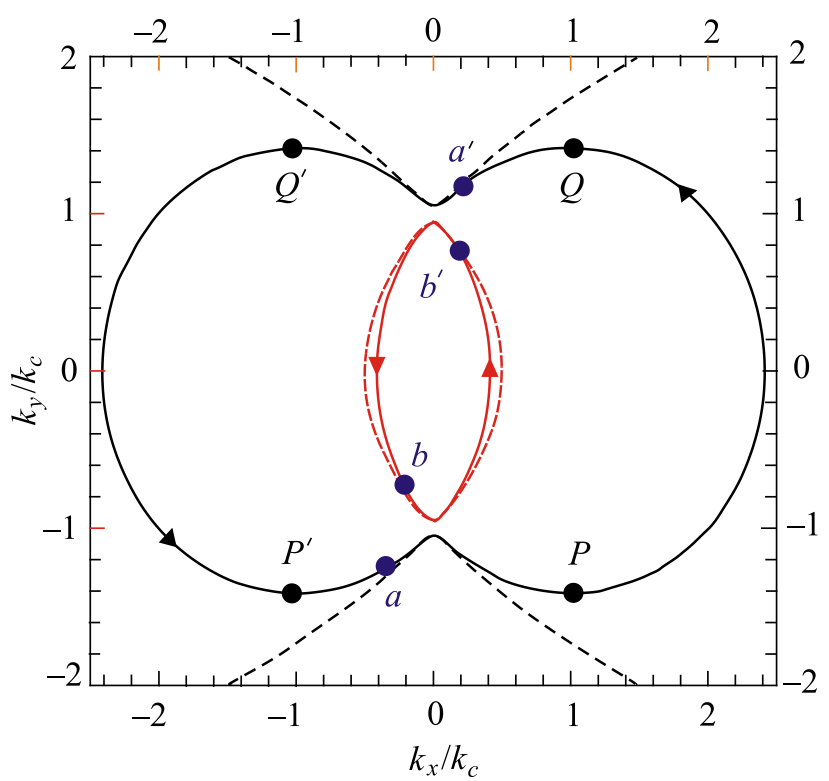

FIG. 3. Effective two-band model. The dashed lines are the approximation Eq. (20) for small $x$. The parameters are $y_{0}=1$ and $\varepsilon_{g}=0.05$. The shape of the small lens, corresponding to the $\alpha$ orbit in magnetic field, is slightly changed by the approximation when $y_{0}$ is small enough. with $\omega(y)=\left(y^{3} / 3-y_{0}^{2} y\right)$ instead of $\omega(y)=y^{2}$. The first double integral in Eq. (7) contributing to $t$ in the large field limit and far from the scattering region can be written as

$$
\begin{aligned}
& \int_{-\infty}^{\infty} d y_{1} \int_{-\infty}^{y_{1}} d y_{2} \mathrm{e}^{i \omega\left(y_{1}\right) / h+i \omega\left(y_{2}\right) / h} \\
& \quad=\int_{-\infty}^{\infty} d y_{1} \int_{-\infty}^{\infty} d y_{2} \oint \frac{d z \mathrm{e}^{-i \omega\left(y_{1}\right) / h+i \omega\left(y_{2}\right) / h+i z\left(y_{1}-y_{2}\right)}}{2 i \pi(z-i \varepsilon)} .
\end{aligned}
$$

We can define each integral over $y_{1}$ and $y_{2}$ as a function of $z$

$$
h(z)=\int_{-\infty}^{\infty} d y \mathrm{e}^{-i \omega(y) / h+i z y}=2 \pi h^{1 / 3} \mathrm{Ai}\left[-h^{1 / 3}\left(\frac{y_{0}^{2}}{h}+z\right)\right] .
$$

Then using $(z-i \varepsilon)^{-1}=P(1 / z)+i \pi \delta(z)$, one obtains

$$
\begin{aligned}
t \simeq & 1+\frac{\varepsilon_{g}^{2}}{h^{2}}\left[2 \pi^{2} h^{2 / 3} \mathrm{Ai}^{2}\left(-h^{1 / 3} \frac{y_{0}^{2}}{h}\right)\right. \\
& \left.+\frac{1}{2 i \pi} \int_{0}^{\infty} \frac{d z}{z}\left[h^{2}(z)-h^{2}(-z)\right]\right] .
\end{aligned}
$$

This expression is valid at large fields. It contains an imaginary part which is due to the presence of the small a orbit between points $b$ and $b^{\prime}$, with area $S_{\alpha}$, in red in Fig. 3 . Indeed, after tunneling through $a$, the particle can be scattered multiple times around the $\alpha$ orbit, and therefore acquires a phase proportional to $S_{\alpha}$, before exiting trough $a^{\prime}$. In the following we compare the transmission coefficient $T=1 /|t|^{2}$ through the small $\alpha$ orbit to the expression given by the semiclassical relation and numerical results.

\section{A. Semiclassical approximation}

The Hamiltonian (20) leads to the set of differential equations for $g_{1}$ and $g_{2}$

$$
\begin{gathered}
h^{2} g_{1}^{\prime \prime}+i h \omega^{\prime}(y) g_{1}^{\prime}-\varepsilon_{g}^{2} g_{1}=0, \\
h^{2} g_{2}^{\prime \prime}-i h \omega^{\prime}(y) g_{2}^{\prime}-\varepsilon_{g}^{2} g_{2}=0,
\end{gathered}
$$

with $\omega^{\prime}(y)=y^{2}-y_{0}^{2}{ }^{*}$ In Fig. 4, we have represented the numerical solution of Eqs. (21) and (25), in particular the modulus of $\left|g_{1}\right|$ for different values of fields. At large values of $y$, we can approximate Eq. (25) by the equations $i h y^{2} g_{1}^{\prime}$ $-\varepsilon_{g}^{2} g_{1} \simeq 0$ and $i h y^{2} g_{2}^{\prime}+\varepsilon_{g}^{2} g_{2} \simeq 0$, which leads to $g_{1} \simeq$ $\exp \left(i \varepsilon_{g}^{2} /(h y)\right) \sim$ constant, and $g_{2} \simeq \exp \left(-i \varepsilon_{g}^{2} /(h y)\right) \sim$ constant. We have chosen $g_{1}(y \ll-1)=1$ and integrated numerically the first differential equation. On the far right, $y \gg 1$, the constant value is proportional to $\exp \left(h_{0} / h\right)$ $=1 / p^{2}$. Therefore, by computing $t$, we can access to the breakdown field $h_{0}$. The semiclassical approximation $g_{1}(y)=\exp (i S(y) / h)$, where $S$ corresponds physically to an area enclosed by the trajectory, consists in expanding $S(y)$ as a series in $h \ll 1$. In particular, at the leading order in $h$ for small field values, one can write $S=S_{0}+h S_{1}+\cdots$ with

*The solutions of Eq. (25) are actually given by triconfluent Heun functions. $^{23}$ 


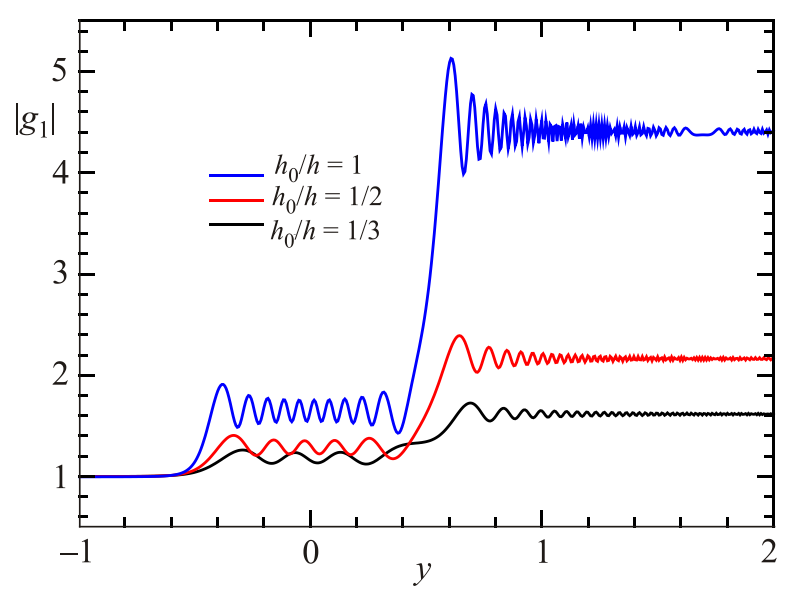

FIG. 4. Wave profile of $g_{1}$ as function of $y$ for three different values of the inverse field ratio $h_{0} / h$. Parameters are $y_{0}=0.5$ and $\varepsilon_{g}=0.02$. From the initial condition $g_{1}(y \ll-1)=1$, we have integrated Eq. (25). The ratio between the two amplitudes $g_{1}(y \gg-1) / g_{1}(y \ll-1)$ is proportional to the inverse of tunneling probability $\exp \left(h_{0} / h\right)=1 / p^{2}$, up to some oscillation factor which corresponds to interferences in the a-pocket (see text). Indeed the electron has to cross two breakdown regions, therefore a factor $p^{2}$ is involved.

$$
\begin{aligned}
& S_{0}^{\prime 2}+\omega^{\prime}(y) S_{0}^{\prime}+\varepsilon_{g}^{2}=0 \\
& S_{0}^{\prime}=\frac{1}{2}\left(-\omega^{\prime}(y) \pm \sqrt{\omega^{\prime}(y)^{2}-4 \varepsilon_{g}^{2}}\right) .
\end{aligned}
$$

When $\omega(y)=y^{2}$, as for the model Eq. (1) (linear sheets of Fig. 2)

$$
S_{0}(y)=-\frac{1}{2} y^{2} \pm \frac{1}{2} y^{2} \sqrt{y^{2}-\varepsilon_{g}^{2}} \mp \frac{1}{2} \varepsilon_{g}^{2} \log \left(y+\sqrt{y^{2}-\varepsilon_{g}^{2}}\right) .
$$

The breakdown field $h_{0}$ is then given by the tunneling amplitude $p=\exp \left(-h_{0} / 2 h\right)$ through the forbidden region, or

$$
h_{0}=2 \int_{-\varepsilon_{g}}^{\varepsilon_{g}} \sqrt{\varepsilon_{g}^{2}-y^{2}}=\varepsilon_{g}^{2} \pi,
$$

which corresponds to the exact result in this particular case. For the second model, Eq. (20) (parabolic sheets of Fig. 3), the breakdown field through one of the two tunneling regions, is instead given by

$$
h_{0}=\int_{\sqrt{y_{0}^{2}-2 \varepsilon_{g}}}^{\sqrt{y_{g}^{2}+2 \varepsilon_{g}}} \sqrt{4 \varepsilon_{g}^{2}-\left(y^{2}-y_{0}^{2}\right)^{2}} d y \simeq \frac{\pi \varepsilon_{g}^{2}}{y_{0}} .
$$

The phase variation of $S_{0}$ around the small pocket corresponds to the area $S_{\alpha}$ of the pocket

$$
\begin{aligned}
S_{\alpha}= & 2 \int_{0}^{\sqrt{y_{0}^{2}-2 \varepsilon_{g}}} \sqrt{\left(y^{2}-y_{0}^{2}\right)^{2}-4 \varepsilon_{g}^{2}} d y \\
= & \frac{4}{3} \sqrt{y_{0}^{2}+2 \varepsilon_{g}}\left[y_{0}^{2} E\left(\sqrt{\frac{y_{0}^{2}-2 \varepsilon_{g}}{y_{0}^{2}+2 \varepsilon_{g}}}\right)\right. \\
& \left.-2 \varepsilon_{g} K\left(\sqrt{\frac{y_{0}^{2}-2 \varepsilon_{g}}{y_{0}^{2}+2 \varepsilon_{g}}}\right)\right] \simeq \frac{4}{3} y_{0}^{3},
\end{aligned}
$$

where $E$ and $K$ are complete elliptic functions of the second and first kind, respectively, and the approximation is taken when $\varepsilon_{g}$ is small. For a unit cell parameter $a=10 \AA$, or, equivalently, a unit cell area of $100 \AA^{2}$, which holds for the organic metals $\theta$-(ET) ${ }_{4} \mathrm{CoBr}_{4}\left(\mathrm{C}_{6} \mathrm{H}_{4} \mathrm{Cl}_{2}\right)$ and $\kappa$-(ET) $\mathrm{Cu}(\mathrm{SCN})_{2}$, the frequency $F_{\alpha}$ and magnetic breakdown field $B_{0}$, expressed in Tesla are given by

$$
\begin{aligned}
& F_{\alpha}=\frac{2 \pi \hbar S_{\alpha}}{a^{2} e}=4136 S_{\alpha}[\mathrm{T}], \\
& B_{0}=\frac{(2 \pi)^{2} \hbar}{a^{2} e} h_{0}=25988 h_{0}[\mathrm{~T}] .
\end{aligned}
$$

As examples, the frequency $F_{\alpha}$ of the two above salts is 944 and $600 \mathrm{~T}$, respectively, yielding $y_{0}=0.55$ and 0.48 . The $\mathrm{MB}$ field $B_{0}=35$ and $16 \mathrm{~T}$, yielding $\varepsilon_{g}=0.015$ and 0.01 , respectively.

\section{B. Transmission coefficient}

We consider the probability of tunneling between points $P$ and $Q$ in Fig. 3, using the model (20), which is defined by the modulus $T=\left|\varphi_{1}(Q) / \varphi_{1}(P)\right|^{2}=1 /|t|^{2}$. Given the approximate value of $t$ in Eq. (24), we can estimate $T$ in the large field limit by exponentiating Eq. (24)

$$
T \simeq \exp \left[-\frac{4 \pi^{2} \varepsilon_{g}^{2}}{h^{4 / 3}} A i^{2}\left(-h^{1 / 3} \frac{y_{0}^{2}}{h}\right)\right] .
$$

$T$ reaches its maximum, or resonance value $T=1$, whenever the Airy function vanishes. This happens when $h=y_{0}^{3}\left(-a_{n}\right)^{3 / 2}$, where $a_{n}<0$ are the zeroes of the Airy functions. For example, $a_{1}=-2.33811, a_{2}=-5.08795$. A comparison with the numerical resolution of the differential equation (21) is shown in Fig. 5. The approximation presents a phase shift more pronounced as the field decreases. Semiclassically, we can compute $T$ using the tunneling matrix (15) between the two points $P$ and $Q$ in Fig. 3. It is the contribution of all possible trajectories between the two points, including the multiple reflections inside the $\alpha$ orbit

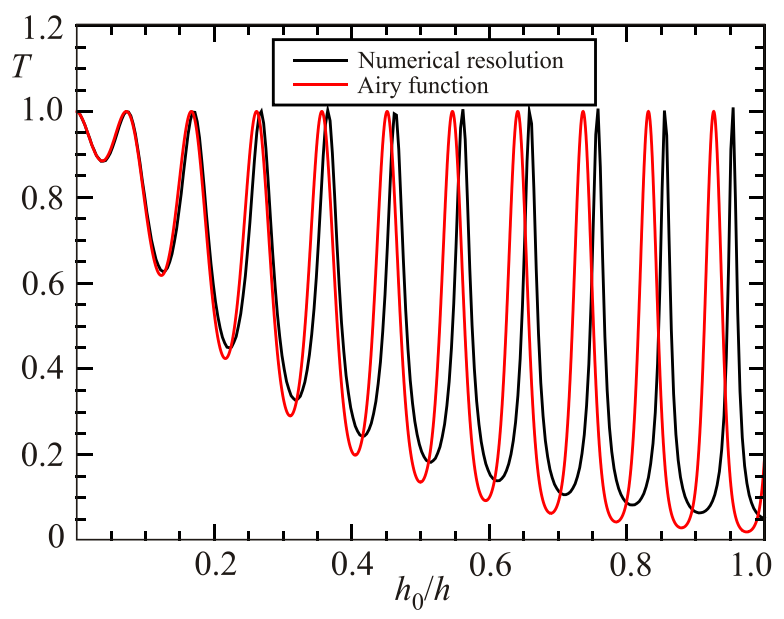

FIG. 5. Transmission coefficient as a function of the inverse field $h_{0} / h$ for $y_{0}=0.5$ and a hybridization coupling $e_{g}=0.02 \quad\left(h_{0}=0.002513\right.$ and $\left.S_{a}=0.159598\right)$. The black line are computed by solving the differential equation (21) and the red line is the large field approximation Eq. (30) obtained by computing approximately $t$ in the S-matrix (24). 


$$
\begin{aligned}
\varphi_{1}(Q)= & \varphi_{1}(P)\left(p i \mathrm{e}^{i S_{\alpha} / 2 h} p\right)+\varphi_{1}(P)\left[p i \mathrm{e}^{i S_{\alpha} / 2 h}\left(-q \mathrm{e}^{-i \phi}\right)\right. \\
& \left.\times i \mathrm{e}^{i S_{\alpha} / 2 h}\left(-q \mathrm{e}^{-i \phi}\right) i \mathrm{e}^{i S_{\alpha} / 2 h} p\right]+\cdots \\
= & \varphi_{1}(P) \frac{i p^{2} \mathrm{e}^{i S_{\alpha} / 2 h}}{1+q^{2} \mathrm{e}^{i S_{\alpha} / h-2 i \phi}} .
\end{aligned}
$$

The factor $i$ corresponds to passing each of the two singular (or turning) points on the surface a Fig. 3 where the slopes are infinite. The phase $\phi$ is taken from Eq. (17). Therefore one obtains (see Ref. 24)

$$
T=\frac{p^{4}}{1+q^{4}+2 q^{2} \cos \left(S_{\alpha} / h-2 \phi\right)} .
$$

$T$ is maximum when the field satisfies $\cos \left(S_{\alpha} / h-2 \phi\right)$ $=-1$, i.e., $T=1$, and the quantized values are given by

$$
h=\frac{S_{\alpha}}{2 \pi n+\pi+2 \phi(h)} .
$$

If $\phi \simeq \pi / 4$, then $h_{0} / h=3 \pi h_{0} / 2 S_{\alpha}, 7 \pi h_{0} / 2 S_{\alpha}, \ldots$ In Fig. 6 is plotted the transmission coefficient as function of the inverse field $h_{0} / h$. The black continuous lines are obtained by solving the system of differential equations (21), with the $g_{1}\left(-y_{c}\right)=1, g_{2}\left(-y_{c}\right)=0, y_{c}=5$, then by computing the ratio $T=1 /|t|^{2}=\left|g_{1}\left(-y_{c}\right) / g_{1}\left(y_{c}\right)\right|^{2}$. Without the phase $\phi$
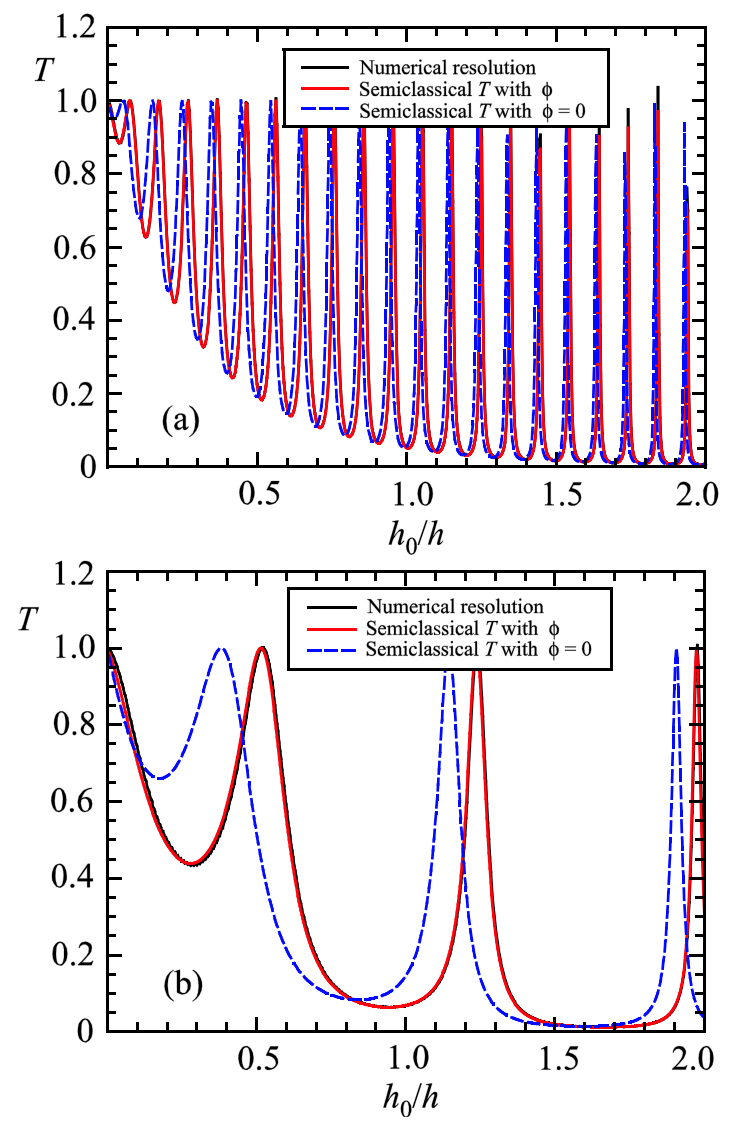

FIG. 6. Transmission coefficient as a function of the inverse field $h_{0} / h$ for $y_{0}=0.5$ and for two values of hybridization coupling: (a) $\varepsilon_{g}=0.02$, $h_{0}=0.002513, S_{\alpha}=0.159598$, and (b) $\varepsilon_{g}=0.05, h_{0}=0.015959$ and $S_{\alpha}=0.131460$. Black lines are computed by solving the differential equation (21). Red lines, which are indiscernible from the black lines, are the result of Eq. (32) where the phase $\phi$ is given by Eq. (17). The dotted lines are obtained without reflection phase $(\phi=0) . \phi=0$ only holds in the limit of small fields $\left(h_{0} / h \gg 1\right)$. from the reflection coefficient (17), the values differ increasingly as the field is increased (dotted blue lines). Oppositely, the phase does not contribute to the oscillations when the field becomes small.

\section{AMPLITUDE RATIOS BETWEEN TWO-INTERACTING ORBITS}

In this section, we consider the model (19), which represents the hybridization of the two giant orbits corresponding to the $\beta$ orbit of the organic metals considered in the last section (see Fig. 1). Using the field quantization, one obtains the set of differential equations

$$
\begin{aligned}
& -h^{2} \partial_{y}^{2} \varphi_{1}+2 i h \partial_{y} \varphi_{1}+\left(y^{2}-y_{0}^{2}\right) \varphi_{2}+2 \varepsilon_{g} \varphi_{2}=0, \\
& 2 \varepsilon_{g} \varphi_{1}-h^{2} \partial_{y}^{2} \varphi_{2}-2 i h \partial_{y} \varphi_{2}+\left(y^{2}-y_{0}^{2}\right) \varphi_{2}=0 .
\end{aligned}
$$

As in preceding sections, we introduce two functions $g_{1}$ and $g_{2}$ such that $\varphi_{i}(y)=g_{i}(y) \exp \left(i \omega_{i}(y) / h\right), \omega_{i}$ are two phase functions that are chosen such that the coefficient of $g_{i}$ vanishes in Eq. (34) after replacement. One obtains

$$
\begin{aligned}
& -h^{2} g_{1}^{\prime \prime}-2 i h\left(\omega_{1}^{\prime}-1\right) g_{1}^{\prime}+2 \varepsilon_{g} g_{2} \exp \left[i\left(\omega_{2}-\omega_{1}\right) / h\right]=0, \\
& -h^{2} g_{2}^{\prime \prime}-2 i h\left(\omega_{2}^{\prime}+1\right) g_{2}^{\prime}+2 \varepsilon_{g} g_{1} \exp \left[i\left(\omega_{1}-\omega_{2}\right) / h\right]=0 .
\end{aligned}
$$

The phase functions satisfy the differential equations

$$
\begin{aligned}
& -i h \omega_{1}^{\prime \prime}+\omega_{1}^{\prime 2}-2 \omega_{1}^{\prime}+y^{2}-y_{0}^{2}=0 \\
& -i h \omega_{2}^{\prime \prime}+\omega_{2}^{\prime 2}+2 \omega_{2}^{\prime}+y^{2}-y_{0}^{2}=0 .
\end{aligned}
$$

We can chose in particular $\omega_{1}=\omega$ and $\omega_{2}=-\bar{\omega}$. The solutions of the Ricatti equations with respect to $\omega^{\prime}$ defined by Eq. (36) can be found in principle using hypergeometric functions. The coefficients $\omega_{1}^{\prime}-1$ and $\omega_{2}^{\prime}+1$ in front of the $g_{i}^{\prime} s$ in Eq. (35) can be removed using an additional transformation $g_{i}^{\prime}(y)=h_{i}(y) \exp \left(2 i \theta_{i}(y) / h\right)$, such that

$$
\theta_{1}=y-\omega_{1}, \quad \theta_{2}=-y-\omega_{2}
$$

Then finally

$$
\begin{aligned}
& h_{1}^{\prime}=\frac{2 \varepsilon_{g}}{h^{2}} g_{2} \mathrm{e}^{-2 i y / h+i\left(\omega_{1}+\omega_{2}\right) / h}, \\
& h_{2}^{\prime}=\frac{2 \varepsilon_{g}}{h^{2}} g_{1} \mathrm{e}^{2 i y / h+i\left(\omega_{1}+\omega_{2}\right) / h} .
\end{aligned}
$$

The whole system can be cast into a system of first-order differential equations

$$
\left(\begin{array}{l}
g_{1}^{\prime} \\
g_{2}^{\prime} \\
h_{1}^{\prime} \\
h_{2}^{\prime}
\end{array}\right)=\left(\begin{array}{cc}
0 & V \\
U & 0
\end{array}\right)\left(\begin{array}{l}
g_{1} \\
g_{2} \\
h_{1} \\
h_{2}
\end{array}\right),
$$

with $U$ and $V$ defined by

$$
\begin{aligned}
& U=\frac{2 \varepsilon_{g} \mathrm{e}^{i\left(\omega_{1}+\omega_{2}\right) / h}}{h^{2}}\left(\begin{array}{cc}
0 & \mathrm{e}^{-2 i y / h} \\
\mathrm{e}^{2 i y / h} & 0
\end{array}\right), \\
& V=\left(\begin{array}{cc}
\mathrm{e}^{2 i y / h-2 \omega_{1} / h} & 0 \\
0 & \mathrm{e}^{-2 i y / h-2 \omega_{2} / h}
\end{array}\right) .
\end{aligned}
$$


The S-matrix can then be formally defined by orderedintegral iterations of the matrix functions $U$ and $V$, similarly as Eq. (4). If we introduce $u(y)=\exp (2 i y / h-2 i \Im(\omega) / h)$ and $v(y)=\exp (2 i y / h-2 i \omega / h)$, one finds that the $t$ matrix element can be expanded as

$$
\begin{aligned}
t= & 1+\frac{4 \varepsilon_{\mathrm{g}}^{2}}{h^{4}} \int_{y \geq y_{1} \geq y_{2} \geq y_{3} \geq y_{4} \geq-y} v\left(y_{1}\right) \bar{u}\left(y_{2}\right) \bar{v}\left(y_{3}\right) u\left(y_{4}\right) \\
& +\frac{16 \varepsilon_{g}^{4}}{h^{8}} \int_{y \geq y_{1} \geq \cdots \geq y_{8} \geq-y} v\left(y_{1}\right) \bar{u}\left(y_{2}\right) \bar{v}\left(y_{3}\right) u\left(y_{4}\right) v\left(y_{5}\right) \\
& \times \bar{u}\left(y_{6}\right) \bar{v}\left(y_{7}\right) u\left(y_{8}\right)+\cdots
\end{aligned}
$$

which is equivalent to Eq. (7) found for one tunneling junction

\section{A. Case with no hybridization $\left(\varepsilon_{g}=0\right)$}

In absence of hybridization, it is interesting to study the phase for an unbounded state (a state where one of the boundary condition for the wave function does not vanish at infinity). The two sheets decouple in this case, and one has only two independent linear second-order differential equations for $g_{1}$ and $g_{2}$. Setting $\varphi_{1}=g_{1}(y) \mathrm{e}^{i y / h}$ and $\varphi_{2}=g_{2}(y)$ $\mathrm{e}^{-i y / h}$, Eq. (34) becomes

$$
\begin{aligned}
& h^{2} g_{1}^{\prime \prime}(y)=\left(y^{2}-r^{2}\right) g_{1}(y), \\
& h^{2} g_{2}^{\prime \prime}(y)=\left(y^{2}-r^{2}\right) g_{2}(y),
\end{aligned}
$$

where $r^{2}=1+y_{0}^{2}$ is the radius of the $\beta$ orbit. It is wellknown that the even and odd solutions are expressed using two Kummer functions $M$ with $y^{2} / h$ as main argument ${ }^{20}$

$$
\begin{aligned}
g_{1}(y) & =A \mathrm{e}^{-y^{2} / 2 h} M\left(\frac{1}{4}-\frac{r^{2}}{4 h}, \frac{1}{2}, \frac{y^{2}}{h}\right)+B y M\left(\frac{3}{4}-\frac{r^{2}}{4 h}, \frac{3}{2}, \frac{y^{2}}{h}\right) \\
& =A u(y)+B v(y) .
\end{aligned}
$$

Solution for the other function $g_{2}$ is similar with independent constants. We impose the constraint that, for large and negative, $g_{1}$ vanishes. This leads to the relation

$$
\frac{B}{2 \Gamma\left(\frac{3}{4}-\frac{r^{2}}{4 h}\right)}-\frac{A}{\sqrt{h} \Gamma\left(\frac{1}{4}-\frac{r^{2}}{4 h}\right)}=0 .
$$

In Fig. 7 (a) is represented $g_{1}$, with a vanishing boundary condition on the left. Only one constant remains, which is not relevant when we consider the ratio of the wave function between $P$ and $Q$ in Fig. 3. Indeed the transmission factor defined here by $T=\left|g_{1}(-r) / g_{1}(r)\right|^{2}$ is exactly equal to

$$
T=\left|\frac{\Gamma\left(\frac{1}{4}-\frac{r^{2}}{4 h}\right) M\left(\frac{1}{4}-\frac{r^{2}}{4 h}, \frac{1}{2}, \frac{r^{2}}{h}\right)+\frac{2 r}{\sqrt{h}} \Gamma\left(\frac{3}{4}-\frac{r^{2}}{4 h}\right) M\left(\frac{3}{4}-\frac{r^{2}}{4 h}, \frac{3}{2}, \frac{r^{2}}{h}\right)}{\Gamma\left(\frac{1}{4}-\frac{r^{2}}{4 h}\right) M\left(\frac{1}{4}-\frac{r^{2}}{4 h}, \frac{1}{2}, \frac{r^{2}}{h}\right)-\frac{2 r}{\sqrt{h}} \Gamma\left(\frac{3}{4}-\frac{r^{2}}{4 h}\right) M\left(\frac{3}{4}-\frac{r^{2}}{4 h}, \frac{3}{2}, \frac{r^{2}}{h}\right)}\right|^{2},
$$

and is a function of $r^{2} / h$. In physical units, the ratio $r^{2} / 2 h$ is equal to the $\beta$-orbit frequency (in Tesla) divided by the magnetic field $B$

$$
\frac{r^{2}}{2 h}=\frac{F_{\beta}}{B}
$$

which is usually a large number $\left(F_{\beta}\right.$ is few thousands of Tesla for organic conductors). It has to be noticed that imposing a vanishing wave function at both negative and positive large values of $y$ (bound state) leads to two conditions

$$
\frac{B}{2 \Gamma\left(\frac{3}{4}-\frac{r^{2}}{4 h}\right)} \pm \frac{A}{\sqrt{h} \Gamma\left(\frac{1}{4}-\frac{r^{2}}{4 h}\right)}=0,
$$

which can only be satisfied when the gamma functions are infinite. This happens when both arguments of the gamma functions are negative integers, and one obtains the usual quantification relation or Landau levels $r^{2}=(2 n+1) h$ with $n$ positive integer. Using the different asymptotic expansions for the Kummer function, ${ }^{1}$ one obtains for each wave function $u$ and $v$ a good approximation near the turning points $y \simeq \pm r$ (see Figs. 7(b) and 7(c), approximation (2))

$$
\begin{aligned}
u(y) \simeq & \sqrt{\pi}\left(\frac{r^{2}}{2 h}\right)^{1 / 6}\left\{\mathrm{Ai}\left[\left(\frac{r^{2}}{2 h}\right)^{2 / 3}\left(\frac{y^{2}}{r^{2}}-1\right)\right] \cos \left(\frac{\pi}{4}-\frac{\pi r^{2}}{4 h}\right)\right. \\
& \left.+\mathrm{Bi}\left[\left(\frac{r^{2}}{2 h}\right)^{2 / 3}\left(\frac{y^{2}}{r^{2}}-1\right)\right] \sin \left(\frac{\pi}{4}-\frac{\pi r^{2}}{4 h}\right)\right\} \\
v(y)= & \frac{\sqrt{\pi}}{2}\left(\frac{r^{2}}{2 h}\right)^{-5 / 6} y\left\{\mathrm{Ai}\left[\left(\frac{r^{2}}{2 h}\right)^{2 / 3}\left(\frac{y^{2}}{r^{2}}-1\right)\right] \cos \left(\frac{3 \pi}{4}-\frac{\pi r^{2}}{4 h}\right)\right. \\
& \left.+\mathrm{Bi}\left[\left(\frac{r^{2}}{2 h}\right)^{2 / 3}\left(\frac{y^{2}}{r^{2}}-1\right)\right] \sin \left(\frac{3 \pi}{4}-\frac{\pi r^{2}}{4 h}\right)\right\} .
\end{aligned}
$$

In the region $-r<y<r$, not too close to the turning points, the solutions are instead adequately approximated by (see Figs. 7(b) and 7(c), approximation (1))

$$
\begin{aligned}
& u(y) \simeq \frac{1}{\sqrt{\sin \theta}}\left[\cos \left(\frac{r^{2}}{2 h}\left(\theta-\frac{1}{2} \sin 2 \theta\right)\right)-\sin \left(\frac{\pi r^{2}}{4 h}\right)\right], \\
& v(y) \simeq-\frac{h}{\sqrt{\sin \theta}}\left[\sin \left(\frac{r^{2}}{2 h}\left(\theta-\frac{1}{2} \sin 2 \theta\right)\right)-\sin \left(\frac{\pi r^{2}}{4 h}\right)\right] .
\end{aligned}
$$



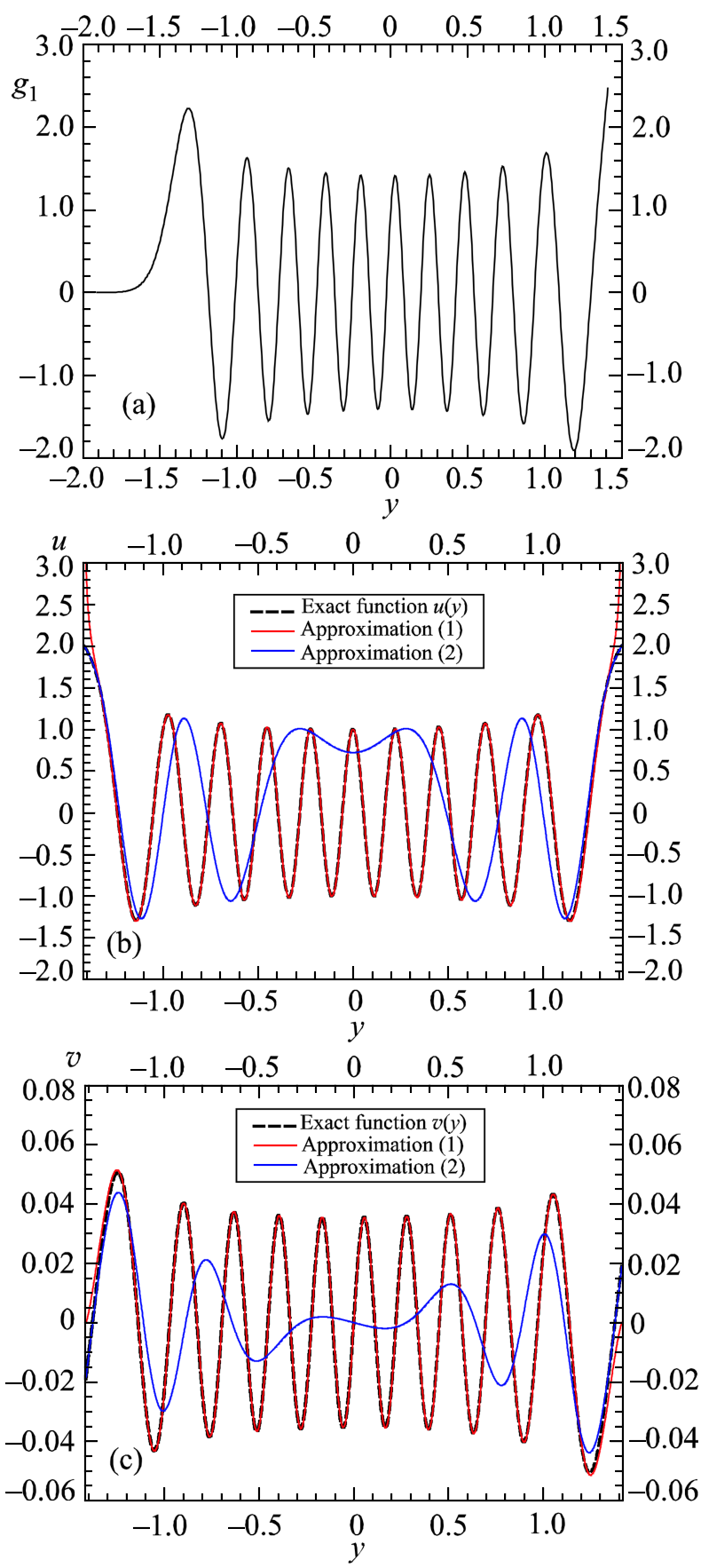

FIG. 7. Wave profile of functions $g_{1}$ (a), $u$ (b), and $v$ (c) as a function of $y$ for field value $h=0.05$ and parameters $y_{0}=1, \quad \varepsilon_{g}=0 \quad\left(r^{2}=2\right)$. Approximation (1) is given by Eqs. (49) and (50), which are accurate in the bulk $-r<y<r$, and approximation (2) by Eqs. (47) and (48), which are correct only near the borders of the turning points $y= \pm r= \pm \sqrt{2}$. Function $g_{1}$ vanishes as $y \rightarrow-\infty$ but is unbounded when $y \rightarrow \infty$.

Moreover, the ratio between the two constants $B$ and $A$ in Eq. (43) is approximated by

$$
\frac{B}{A}=\frac{2 \Gamma\left(\frac{3}{4}-\frac{r^{2}}{4 h}\right)}{\sqrt{h} \Gamma\left(\frac{1}{4}-\frac{r^{2}}{4 h}\right)} \simeq \frac{r}{h} \cot \left(\frac{\pi r^{2}}{4 h}+\frac{\pi}{4}\right) .
$$

Using Eqs. (47) and (48) for $y= \pm r$, and $\operatorname{Bi}(0) / \operatorname{Ai}(0)=\sqrt{3}$, one obtains the semiclassical limit of the inverse transmission factor, and after some algebra and simplifications one obtains the simple result

$$
T \simeq 4 \sin ^{2}\left(\frac{\pi r^{2}}{2 h}+\frac{\pi}{3}\right)=4 \sin ^{2}\left(\pi \frac{F_{\beta}}{B}+\frac{\pi}{3}\right) .
$$

The frequency of the oscillations is $F_{\beta} / 2$ as expected, but there is a shift equal to $\delta=\pi / 3$ as opposed to the semiclassical limit, which is equal to $\delta=\pi / 2$ for a bound state or localized wave function, where at each turning point a Maslov factor equal to $\pi / 2$ is involved after total reflection of the wave function.

\section{B. Semiclassical analysis for interacting orbits}

In this section, one computes semiclassically for a bound state the amplitude ratio between points $P$ and $Q$ in Fig. 3, using a transfer matrix method to obtain all the contributions from the different electronic paths. One has indeed to evaluate the sum of all the amplitudes corresponding to multiple orbits connecting the two points $P$ and $Q$, with their harmonics, and using the connection formula (15) for the tunneling regions. In Fig. 3, we have represented 4 different points (amplitudes) (a, $a^{\prime}, b, b^{\prime}$ ), $a$ and $a^{\prime}$ belong to orbits $\beta$ or $2 \beta-$ $\alpha$, and $b$ and $b^{\prime}$ belong to orbits $\alpha$ or $\beta$. These points are located just before the tunneling event, such that there is a possibility to be transmitted or reflected, just after passing through the breakdown points. A trajectory is an ensemble of steps on the surface, which connect $P$ to $Q$. At time $n=0$ we start from $P$. At later time $n+1$, we can write the amplitudes as function of the amplitudes at time $n$. For example, amplitude $b$ at time $n+1$ is the sum of $b^{\prime}$ after reflection and $a^{\prime}$ after tunneling at time $n$, and can be written as

$$
b(n+1)=p \mathrm{e}^{i S_{\alpha} / 2 h} a^{\prime}(n)-q \mathrm{e}^{i S_{\alpha} / 2 h-i \phi} b^{\prime}(n) .
$$

There are 3 other equations connecting the different points at each step on a trajectory. At $P, Q, P^{\prime}$, and $Q^{\prime}$ we introduce a phase shift $\delta=\pi / 2$. One can write therefore the system

$$
\begin{aligned}
a(n+1)= & q \mathrm{e}^{i\left(S_{\beta}-S_{\alpha} / 2\right) / h+i \phi+2 i \delta} a^{\prime}(n) \\
& +p \mathrm{e}^{i\left(S_{\beta}-S_{\alpha} / 2\right) / h+2 i \delta} b^{\prime}(n), \\
a^{\prime}(n+1)= & q \mathrm{e}^{i\left(S_{\beta}-S_{\alpha} / 2\right) / h+i \phi+2 i \delta} a(n) \\
& +p \mathrm{e}^{i\left(S_{\beta}-S_{\alpha} / 2\right) / h+2 i \delta} b(n), \\
b(n+1)= & -q \mathrm{e}^{i S_{\alpha} / 2 h-i \phi} b^{\prime}(n)+p \mathrm{e}^{i S_{\alpha} / 2 h} a^{\prime}(n), \\
b^{\prime}(n+1)= & -q \mathrm{e}^{i S_{\alpha} / 2 h-i \phi} b(n)+p \mathrm{e}^{i S_{\alpha} / 2 h} a(n) .
\end{aligned}
$$

From these relations, we can define a step matrix $R$, acting on vector $\mathbf{v}(n)^{\mathrm{T}}=\left(a(n), b(n), a^{\prime}(n), b^{\prime}(n)\right)$, with initial condition $\mathbf{v}(0)^{\mathrm{T}}=\left(0,0, \mathrm{e}^{-i\left(S_{\beta}-S_{\alpha} / 2\right) / h-i \delta}, 0\right)$. Then $\mathbf{v}(n+1)=$ $R \mathbf{v}(n)$, with

$$
R=\left(\begin{array}{cc}
0 & A \\
A & 0
\end{array}\right), \quad A=\left(\begin{array}{cc}
q x_{2 \beta-\alpha} \mathrm{e}^{i \phi} & p x_{2 \beta-\alpha} \\
p x_{\alpha} & -q x_{\alpha} \mathrm{e}^{-i \phi}
\end{array}\right),
$$

where $x_{\alpha}=\mathrm{e}^{i S_{\alpha} / 2 h}$ and $x_{2 \beta-\alpha}=\mathrm{e}^{i\left(S_{\beta}-S_{\alpha} / 2\right) / h+2 i \delta}$. We define $T=1 /|t|^{2}=\left|g_{1}(-r) / g_{1}(r)\right|^{2}$ which is also equal to

$$
\begin{aligned}
T^{-1} & =\left|\left\langle\mathbf{v}(0) \mid \mathbf{v}(0)+R^{2} \mathbf{v}(0)+R^{4} \mathbf{v}(0)+\cdots\right\rangle\right|^{2} \\
& =\left|\left\langle\mathbf{v}(0) \mid\left(1-R^{2}\right)^{-1} \mathbf{v}(0)\right\rangle\right|^{2} .
\end{aligned}
$$

Only the even powers of $R$ contribute since to go trough $a^{\prime}$ twice we need to perform an even number of steps. 
Resumming the expression in Eq. (55) involves the inverse of $\left(1-R^{2}\right)$ which can be computed from $\left(1-A^{2}\right)^{-1}$ since $R$ is simply the diagonal block matrix $\operatorname{diag}\left(A^{2}, A^{2}\right)$, and therefore $\left(1-R^{2}\right)^{-1}=\operatorname{diag}\left(\left(1-A^{2}\right)^{-1},\left(1-A^{2}\right)^{-1}\right)$. After some algebra, we extract the third component of $\left(1-R^{2}\right)^{-1} \mathbf{v}(0)$ to obtain $T$

$$
T=\left|\frac{\left(1-x_{\alpha} x_{2 \beta-\alpha}\right)^{2}-q^{2}\left(x_{\alpha} \mathrm{e}^{i \phi}-x_{2 \beta-\alpha} \mathrm{e}^{i \phi}\right)^{2}}{1-p^{2} x_{\alpha} x_{2 \beta-\alpha}-q^{2} x_{\alpha}^{2} \mathrm{e}^{-2 i \phi}}\right|^{2} .
$$

There are two obvious cases. When $p=1$ and $q=0$, one obtains $T=\left|1-x_{\alpha} x_{2 \beta-\alpha}\right|^{2}=\left|1-\mathrm{e}^{i S_{\beta} / h+2 i \delta}\right|^{2}$, or $T=4 \sin ^{2}$ $\left(S_{\beta} / 2 h+\delta\right)$, which was obtained previously in Eq. (52). Oppositely, when $p=0$ and $q=1$, the particle describes orbits around $2 \beta-\alpha$, and $T=\left|1-x_{2 \beta-\alpha}^{2} \mathrm{e}^{2 i \phi}\right|^{2}$, or $T=$ $4 \sin ^{2}\left[\left(S_{\beta}-\frac{1}{2} S_{\alpha}\right) / h+\delta+\phi\right]$. This expression depends on $\phi$ explicitly.

\section{Simple solvable model for two-interacting orbits}

Let us rewrite the Hamiltonian (19) in the representation $\left(x, \hat{y}=-i h \partial_{x}\right)$. One obtains the set of coupled differential equations

$$
\begin{gathered}
-h^{2} \partial_{x}^{2} \partial \varphi_{1}+\left((x+1)^{2}-r^{2}\right) \varphi_{1}+2 \varepsilon_{g} \varphi_{2}=0 \\
2 \varepsilon_{g} \varphi_{1}-h^{2} \partial_{x}^{2} \varphi_{2}+\left((x-1)^{2}-r^{2}\right) \varphi_{2}=0
\end{gathered}
$$

The advantage of this representation is that the imaginary parts in Eq. (34) are absent, at the cost of a shift in the harmonic potential. Function $\varphi_{1}$ is centered around $x=-1$ whereas function $\varphi_{2}$ has dominant weight around $x=1$. We will consider instead a slightly different set of equations

$$
\begin{aligned}
& \left(\hat{y}+\delta_{0}\right)^{2} \varphi_{1}+\left((x+1)^{2}-r^{2}\right) \varphi_{1}+2 \varepsilon_{g}(x) \varphi_{2}=0 \\
& 2 \bar{\varepsilon}_{g}(x) \varphi_{1}+\left(\hat{y}-\delta_{0}\right)^{2} \varphi_{2}+\left((x-1)^{2}-r^{2}\right) \varphi_{2}=0
\end{aligned}
$$

where $\delta_{0}$ is a parameter and the coupling $\varepsilon_{g}$ is a function of $x: \varepsilon_{g}(x)=\left(x-i \delta_{0}\right) g$ with $g$ constant. The Hamiltonian operator $H$ is then defined by

$\hat{H}=\frac{1}{2}\left(\begin{array}{cc}\left(\hat{y}+\delta_{0}\right)^{2}+(x+1)^{2}-r^{2} & 2 g\left(x-i \delta_{0}\right) \\ 2 g\left(x+i \delta_{0}\right) & \left(\hat{y}-\delta_{0}\right)^{2}+(x-1)^{2}-r^{2}\end{array}\right)$,

and the Fermi surface is the location of points given by the equation

$$
\begin{aligned}
H(x, y)= & \frac{1}{4}\left[\left(y+\delta_{0}\right)^{2}+(x+1)^{2}-r^{2}\right] \\
& \times\left[\left(y-\delta_{0}\right)^{2}+(x-1)^{2}-r^{2}\right]-g^{2}\left(x^{2}+\delta_{0}^{2}\right)=0 .
\end{aligned}
$$

For $g$ and $\delta_{0}$ non-zero, the surface is composed of two sheets separated by a gap proportional to $\delta_{0}$, see Fig. 8(a). It has to be noticed that for this particular choice of coupling function, there is no observable gap on the Fermi surface when $\delta_{0}=0$, since $\varepsilon_{g}(0)=0$, but the two surfaces are still coupled at other points by $g x \neq 0$, see Fig. 8(b). The advantage of the Hamiltonian (59) is that it can be factorized using simple
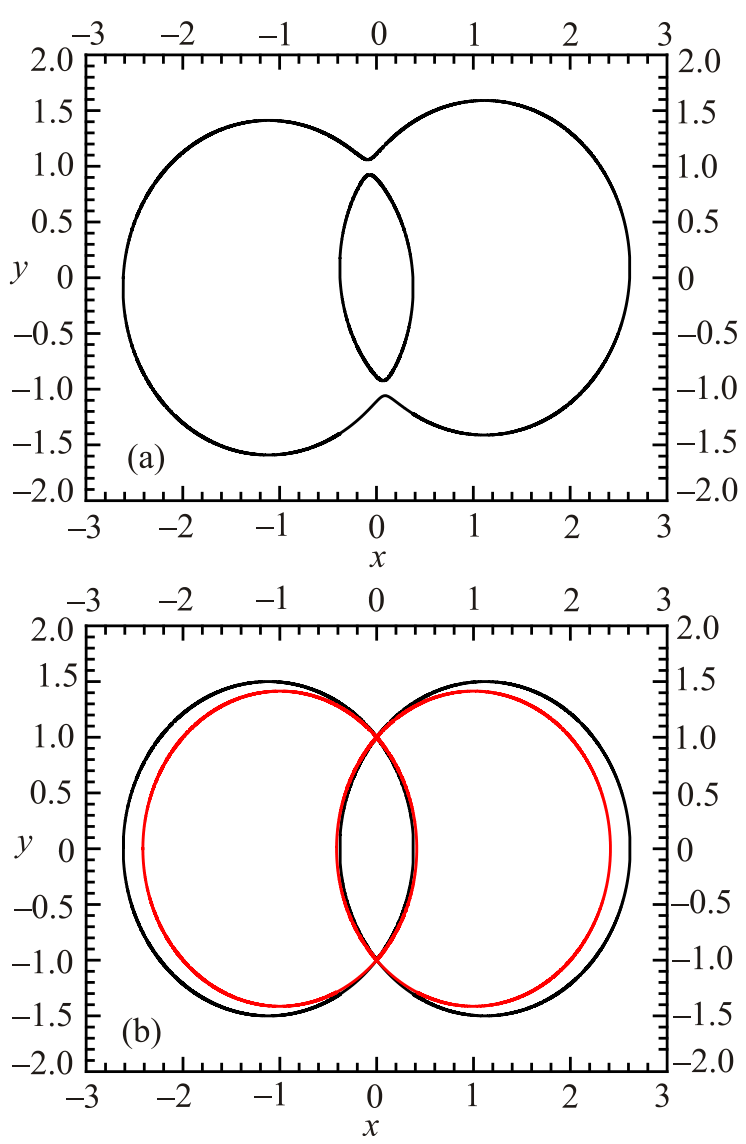

FIG. 8. (a) Fermi surface for $g=0.5$ and $\delta_{0}=0.1$, where a gap is present. The two surfaces are tilted as their centers are not aligned on the horizontal axis. (b) Fermi surface for $g=0.5$ and $\delta_{0}=0$ (black), and $g=\delta_{0}=0$ (red). When $g \neq 0$, the area of the circular cyclotronic trajectories is slightly larger since it is proportional to $r^{2}+g^{2}$.

bosonic operators associated with centers $\pm\left(1 \pm i \delta_{0}\right)$ in the complex plane $(x, y)$

$$
\begin{aligned}
a & =\frac{1}{\sqrt{2 h}}\left(x+1+i \delta_{0}+h \partial_{x}\right), \\
a^{\dagger} & =\frac{1}{\sqrt{2 h}}\left(x+1-i \delta_{0}-h \partial_{x}\right), \\
b & =\frac{1}{\sqrt{2 h}}\left(x-1-i \delta_{0}+h \partial_{x}\right), \\
b^{\dagger} & =\frac{1}{\sqrt{2 h}}\left(x-1+i \delta_{0}-h \partial_{x}\right),
\end{aligned}
$$

with $\left[a, a^{\dagger}\right]=\left[b, b^{\dagger}\right]=1$. The set of differential equations (58) are indeed identical to two coupled harmonic oscillators

$$
\begin{aligned}
h\left(a^{\dagger} a+\frac{1}{2}\right) \varphi_{1}+\varepsilon_{g} \varphi_{2} & =\frac{r^{2}}{2} \varphi_{1}, \\
h\left(b^{\dagger} b+\frac{1}{2}\right) \varphi_{2}+\bar{\varepsilon}_{g} \varphi_{1} & =\frac{r^{2}}{2} \varphi_{2},
\end{aligned}
$$

and it is straightforward then to consider the following twodimensional "bosonic" operators:

$$
P=\left(\begin{array}{cc}
a & \frac{1}{\sqrt{2 h}} \\
\frac{g}{\sqrt{2 h}} & b
\end{array}\right), \quad P^{\dagger}=\left(\begin{array}{cc}
a^{\dagger} & \frac{g}{\sqrt{2 h}} \\
\frac{g}{\sqrt{2 h}} & b^{\dagger}
\end{array}\right)
$$


to express the Hamiltonian as an extended harmonic oscillator in two-dimensions

$\hat{H}\left(\begin{array}{l}\varphi_{1} \\ \varphi_{2}\end{array}\right)=\left\{h P^{\dagger} P+\frac{1}{2}\left(\begin{array}{cc}h-r^{2}-g^{2} & 0 \\ o & h-r^{2}-g^{2}\end{array}\right)\right\}\left(\begin{array}{l}\varphi_{1} \\ \varphi_{2}\end{array}\right)=0$.

The "bosonic" operators $P$ and $P^{\dagger}$ satisfy the commutation relation

$$
\left[P, P^{\dagger}\right]=Q_{0}=\left(\begin{array}{cc}
1 & 2 i g \delta_{0} / h \\
-2 i g \delta_{0} / h & 1
\end{array}\right)=\sigma_{0}-2 g \delta_{0} \sigma_{2} / h,
$$

which is not unity when the product $g \delta_{0}$ is not zero. We cannot therefore call them "bosonic" in the usual sense since there is a mixing of the two different types of bosons due to the coupling. Here $\sigma_{i=0.3}$ are the usual Dirac matrices in two dimensions. ${ }^{*}$ There are two possible ways to construct the wave functions, depending on the value of $\delta_{0}$. If $\delta_{0}=0$, then $P$ and $P^{\dagger}$ are true bosonic operators, and we can construct the ground-state solution $P \Psi_{0}=0$ of lowest energy $E_{0}$ $=\frac{1}{2}\left(h-r^{2}-g^{2}\right)=0$, with $\Psi_{0}=\left(\varphi_{1}^{(0)}, \varphi_{2}^{(0)}\right)^{\mathrm{T}} / \sqrt{2}$. This imposes the constraint $h=r^{2}+g^{2}$ on the field. Normally we construct the states above the ground-state energy by quantization of the area, or $E_{n}=h\left(n+\frac{1}{2}\right) \propto r^{2}+g^{2}$, but here we keep $r$ constant (or constant Fermi energy) and solve for $h$ values for which a set of bounded wave functions can be found. It is easy to see that the first component $\varphi_{1}^{(0)}$ satisfies the factorized differential equation

$$
\left(x+h \partial_{x} \pm \sqrt{1+g^{2}}\right)\left(x+h \partial_{x} \mp \sqrt{1+g^{2}}\right) \varphi_{1}^{(0)}=0 .
$$

The solutions are simple combinations of two Gaussian exponentials centered at $\pm x_{g}= \pm \sqrt{1+g^{2}}$

$$
\begin{aligned}
\varphi_{1}^{(0)}(x)= & A \exp \left[-\frac{\left(x+x_{g}\right)^{2}}{2 h}\right]+B \exp \left[-\frac{\left(x-x_{g}\right)^{2}}{2 h}\right], \\
\varphi_{2}^{(0)}(x)= & -\frac{1-\sqrt{1+g^{2}}}{g} A \exp \left[-\frac{\left(x+x_{g}\right)^{2}}{2 h}\right] \\
& -\frac{1+\sqrt{1+g^{2}}}{g} B \exp \left[-\frac{\left(x-x_{g}\right)^{2}}{2 h}\right] .
\end{aligned}
$$

The two components are coupled together once the constants $A$ and $B$ are determined. These constants satisfy a conservation equation, depending on the filling factor. If we consider initially a system filled with one electron in each orbital at zero coupling, therefore two electrons in total, we impose that, by increasing the coupling, the number of electrons per orbital does not change. One has the pair of constraints $\int\left|\varphi_{1}^{(0)}\right|^{2}=\int\left|\varphi_{2}^{(0)}\right|^{2}=1$ (in this case we consider real functions), which leads to $\left\langle\Psi_{0} \mid \Psi_{0}\right\rangle=1$, and to the following relations of conservation:

*We remind that the Dirac matrices are defined by $\sigma_{0}=\left(\begin{array}{ll}1 & 0 \\ 0 & 1\end{array}\right)$, $\sigma_{1}=\left(\begin{array}{ll}0 & 1 \\ 1 & 0\end{array}\right), \sigma_{2}=\left(\begin{array}{cc}0 & -i \\ i & 0\end{array}\right)$, and $\sigma_{3}=\left(\begin{array}{cc}1 & 0 \\ 0 & -1\end{array}\right)$. $\frac{1}{\sqrt{\pi h}}=A^{2}+B^{2}+2 A B \mathrm{e}^{-x_{g}^{2} / h}$,

$\frac{1}{\sqrt{\pi h}}=A^{2}\left(\frac{1-\sqrt{1+g^{2}}}{g}\right)^{2}+B^{2}\left(\frac{1+\sqrt{1+g^{2}}}{g}\right)^{2}-2 A B \mathrm{e}^{-x_{g}^{2} / h}$.

The other state vectors at higher energy (or higher nodes) are given by the successive application of on $P^{\dagger}$ on $\Psi_{0}$

$$
\Psi_{n}=\frac{1}{\sqrt{2}}\left(\begin{array}{c}
\varphi_{1}^{(n)} \\
\varphi_{2}^{(n)}
\end{array}\right)=\frac{1}{\sqrt{n !}} P^{\dagger} \Psi_{0}
$$

with energy $E_{n}=h\left(n+\frac{1}{2}\right)-\left(r^{2}+g^{2}\right) / 2$. When $E_{n}=0$, this imposes a field value $h_{n}=\left(r^{2}+g^{2}\right) /(2 n+1)$ for which $\Psi_{n}$ is solution of Eq. (57). In Fig. 9, we have represented the two components $\varphi_{1}^{(n)}$ and $\varphi_{2}^{(n)}$ for the state $n=10$ at constant $r^{2}=2$. In the limit of small coupling, Eq. (68) leads to the solutions (we choose $A>0$ and $B<0$ )

$$
\begin{aligned}
A & \simeq(\pi h)^{-1 / 4}, \quad B \simeq-\frac{g}{2}(\pi h)^{-1 / 4} \rightarrow 0, \\
\varphi_{1}^{(0)} & \simeq(\pi h)^{-1 / 4} \exp \left[-\frac{\left(x+x_{g}\right)^{2}}{2 h}\right], \\
\varphi_{2}^{(0)} & \simeq(\pi h)^{-1 / 4} \exp \left[-\frac{\left(x+x_{g}\right)^{2}}{2 h}\right],
\end{aligned}
$$
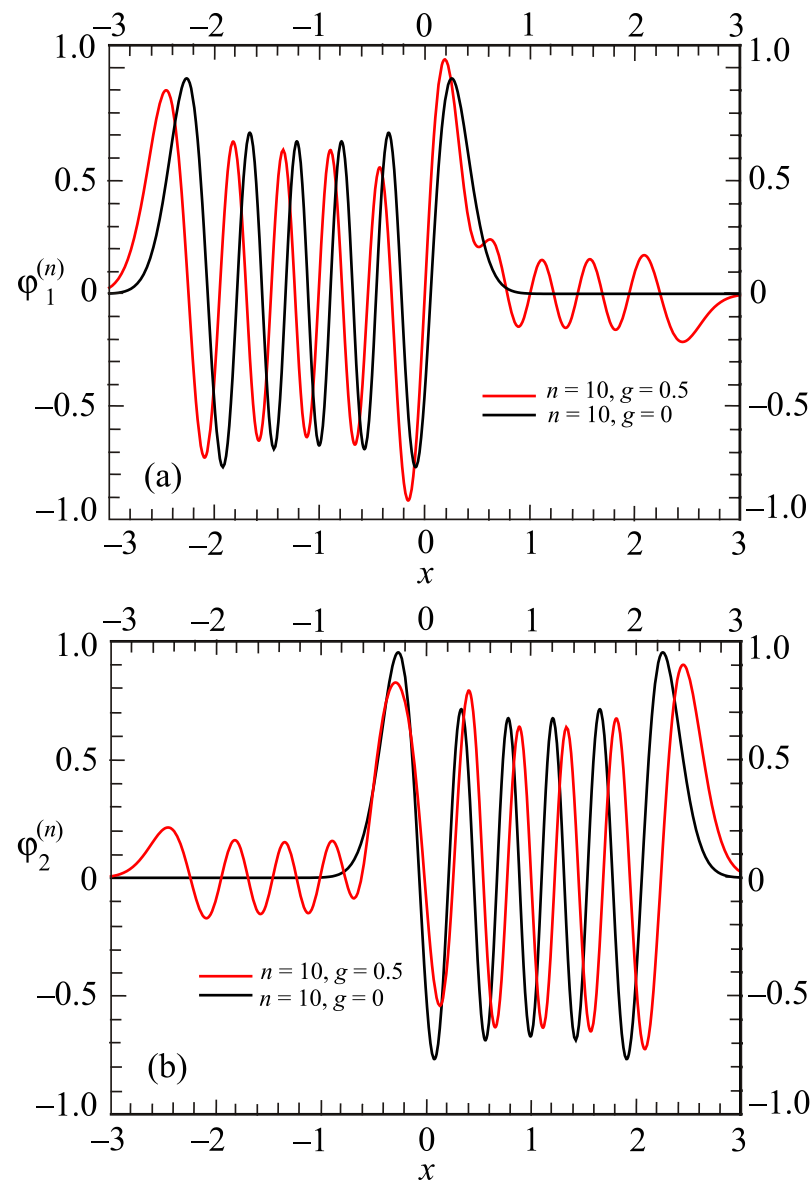

FIG. 9. Wave profile of bound states $\varphi_{1}^{(n)}$ and $\varphi_{2}^{(n)}$ for coupling parameters $g=0.5$ and $\delta_{0}=0$ (red), at level $n=10$, and comparison with the free case ( $g=0$ (black), independent harmonic oscillators). For $g=0.5$ and $g=0$, we take $h=\left(r^{2}+g^{2}\right) /(2 n+1)$, corresponding to $h=0.107$ and $h=0.095$, respectively. Constant $A=(\pi h)^{1 / 4}$, and $B$ is deduced from Eq. (68). 
which is expected for two independent orbitals. In general, the two constants $A$ and $B$ are not independent because of Eq. (68), which leads to an effective coupling between the two components of the wave function.

Let us now consider the case $\delta_{0} \neq 0$. The ground state is still defined by $P \Psi_{0}=0$. Setting $z_{g}=\sqrt{\left(1+i \delta_{0}\right)^{2}+g^{2}}$, one obtains

$$
\begin{aligned}
\varphi_{1}^{(0)}(x)= & A \exp \left[-\frac{\left(x+z_{g}\right)^{2}}{2 h}\right]+B \exp \left[-\frac{\left(x-z_{g}\right)^{2}}{2 h}\right], \\
\varphi_{2}^{(0)}(x)= & -\frac{1+i \delta_{0}-z_{g}}{g} A \exp \left[-\frac{\left(x+z_{g}\right)^{2}}{2 h}\right] \\
& -\frac{1+i \delta_{0}+z_{g}}{g} B \exp \left[-\frac{\left(x-z_{g}\right)^{2}}{2 h}\right] .
\end{aligned}
$$

The conditions of normalization are the same as before, which leads to a set of complex equations similar to Eq. (68). The commutator (65) prevents us to construct the excited states $\Psi_{n}$, which satisfies $P^{\dagger} P \Psi_{n}=n \Psi_{n}$, directly from successive applications of $P^{\dagger}$ on the ground state. Instead we have to seek for linear combinations of functions $P^{\dagger n} \Psi_{0}$

$$
\Psi_{n}=R_{n}^{(n)} P^{\dagger n} \Psi_{0}+R_{n-1}^{(n)} P^{\dagger n-1} \Psi_{0}+\cdots+R_{(0)}^{(n)} \Psi_{0},
$$

where $R_{k}^{(n)}$ are constant matrices to be determined selfconsistently. In the limit $\delta_{0} \rightarrow 0$, only the matrix $R_{n}^{(n)}$ does not vanish, and corresponds to the normalization factor. Computing $P^{\dagger} P \Psi_{n}=n \Psi_{n}$ leads to a set of $(n+1)$ relations between these matrices at order $n$. In particular, by application of $P^{\dagger} P$ on each element of Eq. (72), one has

$$
\begin{aligned}
P^{\dagger} P R_{k}^{(n)} P^{\dagger k} \Psi_{0}= & \left(\left[P^{\dagger},\left[P, R_{k}^{(n)}\right]\right]-R_{k}^{(n)} Q_{0}\right) P^{\dagger k} \Psi_{0} \\
& +\left[P, R_{k}^{(n)}\right] P^{\dagger k+1} \Psi_{0}+\left[P^{\dagger}, R_{k}^{(n)}\right] P P^{\dagger k} \Psi_{0} \\
& +R_{k}^{(n)} P P^{\dagger k+1} \Psi_{0} .
\end{aligned}
$$

For the last two terms, after some algebra, we can move the operator $P$ to the right of $P^{\dagger k}$ and $P^{\dagger k+1}$ using the binomial relation

$$
\begin{aligned}
P P^{\dagger k} \Psi_{0} & =\sum_{i=0}^{k-1}\left(\begin{array}{c}
k \\
1
\end{array}\right) Q_{k-1-l} P^{\dagger l} \Psi_{0}, \\
Q_{l} & =\left[P^{\dagger}, Q_{l-1}\right],\left[P, P^{\dagger}\right]=Q_{0} .
\end{aligned}
$$

The matrices $Q_{k}$ are zero when $Q_{0}=1$, and in this case we have simply $P P^{\dagger n} \Psi_{0}=n P^{\dagger n-1} \Psi_{0}$. The identification of each coefficient of $P^{\dagger k} \Psi_{0}$ in the equation $P^{\dagger} P \Psi_{n}=n \Psi_{n}$ leads to the set of $(n+1)$ equations which are composed of commutators. In particular, the first three equations read

$$
\begin{aligned}
& {\left[P, R_{n}^{(n)}\right]=0, \quad\left[P, R_{n-1}^{(n)}\right]+n R_{n}^{(n)}\left(Q_{0}-1\right)=0,} \\
& {\left[P, R_{n-2}^{(n)}\right]+R_{n-1}^{n}\left[(n-1) Q_{0}-n\right]+\left[P^{\dagger},\left[P, R_{n-1}^{(n)}\right]\right]} \\
& \quad+n\left[P^{\dagger}, R_{n}^{(n)}\right] Q_{0}+\frac{1}{2} n(n+1) R_{n}^{(n)} Q_{1}=0 .
\end{aligned}
$$

This can be solved, for example, using Dirac matrices with unknown scalar coefficients. For example, the matrix coefficients of the first excited state $n=1, \Psi_{1}=\left(R_{1} P^{\dagger}+R_{0}\right) \Psi_{0}$, can be found by solving the two equations

$$
\left[P, R_{1}\right]=0, \quad\left[P, R_{0}\right]=R_{1}\left(1-Q_{0}\right)
$$

It is useful to write $P$ and $P^{\dagger}$ using $2 \times 2$ Dirac matrices

$$
\begin{aligned}
P & =\frac{1}{\sqrt{2 h}}\left[\left(x+h \partial_{x}\right) \sigma_{0}+g \sigma_{1}+\left(1+i \delta_{0}\right) \sigma_{3}\right], \\
P^{\dagger} & =\frac{1}{\sqrt{2 h}}\left[\left(x-h \partial_{x}\right) \sigma_{0}+g \sigma_{1}+\left(1-i \delta_{0}\right) \sigma_{3}\right],
\end{aligned}
$$

and separate the part proportional to identity from the remaining $\sigma_{I}$ 's $: P=(2 h)^{-1 / 2}\left(x+h \partial_{x}\right) \sigma_{0}+P_{0}=D+P_{0}$ and $P^{\dagger}=(2 h)^{-1 / 2}\left(x-h \partial_{x}\right) \sigma_{0}+P_{0}^{\dagger}=D^{\dagger}+P_{0}^{\dagger}$, with constant matrices

$$
\begin{aligned}
& P_{0}=\frac{1}{\sqrt{2 h}}\left(\begin{array}{cc}
1+i \delta_{0} & \varepsilon_{g} \\
\varepsilon & -1-i \delta_{0}
\end{array}\right), \\
& P_{0}^{\dagger}=\frac{1}{\sqrt{2 h}}\left(\begin{array}{cc}
1-i \delta_{0} & \varepsilon_{g} \\
\varepsilon & -1+i \delta_{0}
\end{array}\right),
\end{aligned}
$$

and $\left[D, D^{\dagger}\right]=\sigma_{0}$. Differential operators $D$ and $D^{\dagger}$ are proportional to the identity matrix and commute with $P_{0}$ and $P_{0}^{\dagger}$ which are constant matrices. Then the solutions of Eq. (75) can be expressed using $P_{0}$ and $P_{0}^{\dagger}$ only. An obvious solution of the first equation is $R_{1}=-\alpha_{0} \sigma_{0}+\alpha_{1} P_{0} P_{0}^{\dagger}$, where $\alpha_{0}$ and $\alpha_{1}$ are constants which are determined by orthogonality and normalization of the wave functions $\Psi_{0}$ and $\Psi_{1}$. Then a solution of the second equation is simply $R_{1}=-\left(\alpha_{0} \sigma_{0}+\alpha_{1} P_{0}\right) P_{0}^{\dagger}$. In particular, this leads to the factorization

$$
\Psi_{1}=\left(\alpha_{0} \sigma_{0}+\alpha_{1} P_{0}\right)\left(P^{\dagger}-P_{0}^{\dagger}\right) \Psi_{0}=\left(\alpha_{0} \sigma_{0}+\alpha_{1} P_{0}\right) D^{\dagger} \Psi_{0} .
$$

Writing the condition $\left\langle\Psi_{0} \mid \Psi_{1}\right\rangle=0$ leads to

$$
\alpha_{0}\left\langle\Psi_{0} \mid P_{0}^{\dagger} \Psi_{0}\right\rangle+\alpha_{1}\left\langle\Psi_{0} \mid P_{0}^{\dagger} P_{0} \Psi_{0}\right\rangle=0
$$

The normalization $\left\langle\Psi_{1} \mid \Psi_{1}\right\rangle=1$ gives a supplementary condition which fixes the two constants (up to a phase factor)

$$
\begin{aligned}
\left\langle P_{0} P_{0}^{\dagger}\right\rangle^{2}= & \left|\alpha_{0}\right|^{2}\left(\left\langle P_{0}^{\dagger} P_{0}\right\rangle\left(1+\left\langle P_{0}^{\dagger} P_{0}\right\rangle\right)-\left(\left\langle P_{0}\right\rangle^{2}+\left\langle P_{0}^{\dagger}\right\rangle^{2}\right)\right. \\
& \times\left\langle P_{0}^{\dagger} P_{0}\right\rangle-\left\langle P_{0}^{\dagger} P_{0}\right\rangle\left(\left\langle P_{0}\right\rangle\left\langle P_{0} P_{0}^{\dagger} P_{0}\right\rangle+\left\langle P_{0}^{\dagger}\right\rangle\left\langle P_{0}^{\dagger 2} P_{0}\right\rangle\right) \\
& \left.-\left\langle P_{0}\right\rangle\left\langle P_{0}^{\dagger}\right\rangle\left\langle\left(P_{0}^{\dagger} P_{0}\right)^{2}\right\rangle\right),
\end{aligned}
$$

where we have omitted $\Psi_{0}$ in the scalar products to simplify the notations. When no coupling is present $\varepsilon_{g}=0, P_{0}$ $=\left(1+i \delta_{0}\right) \sigma_{3}$, and $P_{0}^{\dagger} P_{0}=P_{0} P_{0}^{\dagger}=\left(1+\delta_{0}^{2}\right) \sigma_{0}$. We also assume that in this case that $\left\langle P_{0}\right\rangle=\left\langle P_{0}^{\dagger}\right\rangle=0$, so that $\left|\alpha_{0}\right|^{2}$ $=2$ and $\alpha_{1}=0$, which corresponds to the uncoupled model of two electrons in two independent orbits. This method allows for the construction of all excited states and can be generalized for a linear chain of $N$ coupled orbits. Indeed we can represent the $P$ and $P^{\dagger}$ operators as extended matrix operators of dimension $N$ with coupling parameters $g$ and $\delta_{0}$ similar to Eq. (63), and centers corresponding to each individual oscillator. For example, in Fig. 10, we have represented such surface, for $N=4$ connected orbits, by considering the following extended bosonic operators in four dimensions 


$$
P=\frac{1}{\sqrt{2 h}}\left(\begin{array}{cccc}
x+3+i \delta_{0}+h \partial_{x} & g & 0 & 0 \\
g & x+1-i \delta_{0}+h \partial_{x} & g & 0 \\
0 & g & x-1+i \delta_{0}+h \partial_{x} & g \\
0 & 0 & g & x-3-i \delta_{0}+h \partial_{x}
\end{array}\right)
$$

and

$$
P^{\dagger}=\frac{1}{\sqrt{2 h}}\left(\begin{array}{cccc}
x+3-i \delta_{0}-h \partial_{x} & g & 0 & 0 \\
g & x+1+i \delta_{0}-h \partial_{x} & g & 0 \\
0 & g & x-1-i \delta_{0}-h \partial_{x} & g \\
0 & 0 & g & x-3+i \delta_{0}-h \partial_{x}
\end{array}\right) .
$$

\section{ONSAGER PHASE OF DE HAAS-VAN ALPHEN OSCILLATIONS IN LINEAR CHAINS OF COUPLED ORBITS}

In this section, we consider de Haas-van Alphen oscillations observed in quasi-two-dimensional organic metals with a Fermi surface which can be regarded as a linear chain of orbits coupled by magnetic breakdown. Recall that Fourier spectra of these compounds is composed of Fourier components, labeled $\eta$ in the following, the frequency of which are linear combinations of that linked to the closed orbit $\alpha$ and the magnetic breakdown orbit $\beta: F_{\eta}=n_{\alpha} F_{\alpha}+n_{\beta} F_{\beta}$. The field- and temperature-dependent amplitude of several of these components does not follow the usual Lifshitz-Kosevich formula due to oscillation of the chemical potential in magnetic field. Nevertheless, Fourier amplitudes are accounted for by a development up to the second order in damping factors in this case. $^{10,15,16}$ An extensive discussion of this problematic is given in Refs. 25 and 26. As an example, let us consider magnetic torque data relevant to the organic metal $\theta$-(ET) $\mathrm{CoBr}_{4}\left(\mathrm{C}_{6} \mathrm{H}_{4} \mathrm{Cl}_{2}\right)$. Field- and temperature-dependent de Haasvan Alphen oscillations amplitudes of this organic metal are consistently accounted for by this formalism with the following parameters: $F_{\alpha}=(944 \pm 4) \mathrm{T}, F_{\beta}=(4600 \pm 10) \mathrm{T}, \quad m_{\alpha}=$ $1.81 \pm 0.05, m_{\beta}=3.52 \pm 0.19, \quad g_{\alpha}^{*}=g_{\beta}^{*}=1.9 \pm 0.2, \quad T_{D \alpha}=T_{D \beta}$ $=(0.79 \pm 0.10) \mathrm{K}, B_{0}=(35 \pm 5) \mathrm{T}$, where $F_{\alpha(\beta)}, m_{\alpha(\beta)}, g_{\alpha(\beta)}^{*}$, $T_{D \alpha(\beta)}$, and $B_{0}$ are the frequencies, effective masses, effective Lande factors, Dingle temperatures and magnetic breakdown field, respectively. ${ }^{15}$ Furthermore, the Onsager phase of the various Fourier components is accounted for by Eq. (17), yielding ${ }^{10}$

$$
\phi_{\eta}=\phi_{\eta}-n_{\eta}^{r} \phi(B)
$$

where $n_{\eta}^{r}$ is the number of reflections events and $\varphi_{\eta}$ is equal to $\pi / 2$ times the number of turning points of the $\eta$ orbit. De

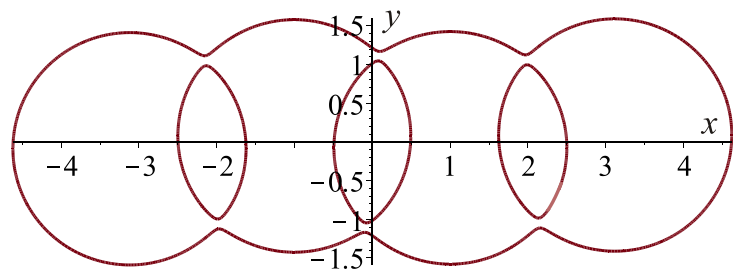

FIG. 10. Fermi surface of four individual coupled orbits, constructed from operators (80) and (81), with coupling parameters $g=0.5$ and $\delta_{0}=0.1$.
Haas-van Alphen oscillations of Fig. 11 are obtained with this set of parameters, except that various values of $B_{0}$ are explored. As expected, as $B_{0}$, hence the reflection probability $q$, increases, the amplitude of all the components involving $\beta$ decreases and, at very high $B_{0}$, only remain the contributions of $\alpha$ and its harmonics. The striking point, on which we will focus in the following, is the observed shift of the $\alpha$ oscillations, for which $n_{\alpha}^{r}=2,{ }^{25,26}$ as $B_{0}$ varies (whereas the Onsager phase of $\beta$ oscillation remains unchanged since $n_{\beta}^{r}=0^{10}$ ).

Strictly speaking, the oscillations are not periodic in $1 / B$ for finite $B_{0}$ values. This effect can be quantified considering an "apparent frequency" $F_{\text {app }}=1 /\left(B_{i}^{-1}-B_{i+1}^{-1}\right)$, where the indexes $i$ and $i+1$ mark two successive oscillation maxima.

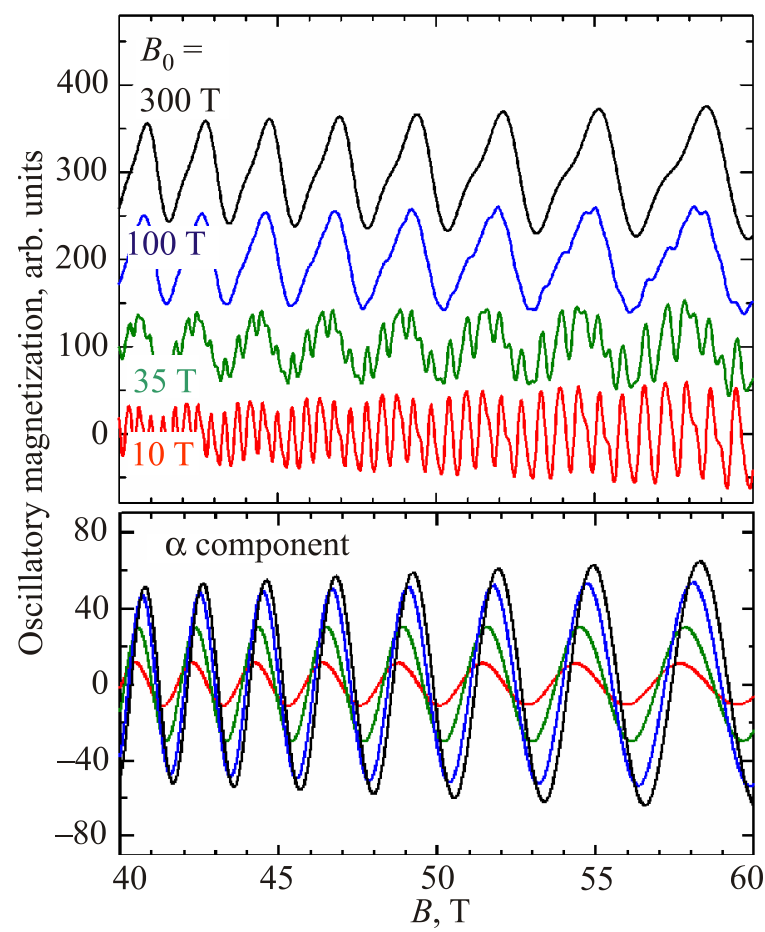

FIG. 11. (a) De Haas-van Alphen oscillations calculated with the parameters (effective masses, Dingle temperature, etc.) relevant to $\theta-(\mathrm{ET})_{4} \mathrm{CoBr}_{4}$ $\left(\mathrm{C}_{6} \mathrm{H}_{4} \mathrm{Cl}_{2}\right)^{15}$ albeit for various values of the magnetic breakdown field $B_{0}$ ( $B_{0}=35 \mathrm{~T}$ holds for the experimental data). Contribution of the component a is given in (b): as $B_{0}$ increases, its amplitude increases and the On-sager phase shifts towards high fields. 


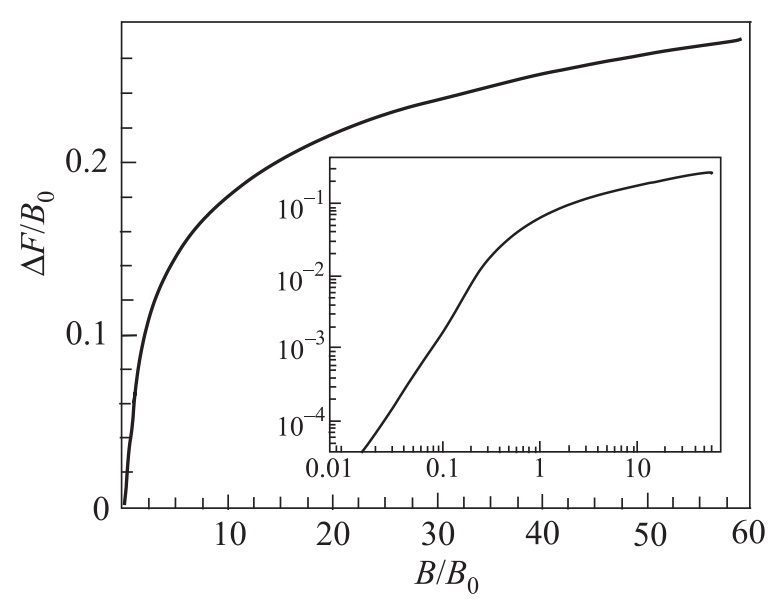

FIG. 12. Field dependence of the "apparent frequency" predicted by Eq. (83) for $n_{\eta}^{r}=2$ which stands for $\alpha$ oscillations of the linear chain of coupled orbits.

According to Eq. (82), $F_{\text {app }}=F_{\eta}+\left(B_{0} / 4 \pi^{2}\right) d \phi / d u$, yielding an "universal" frequency shift

$$
\frac{\Delta F}{B_{0}}=\frac{1}{4 \pi^{2}} \frac{d \phi_{\eta}}{d u}
$$

where $\Delta F=F_{\text {app }}-F$, which depends on $u$ (see Eq. (17)), i.e., on the ratio $B / B_{0}$, only for a given $n_{\eta}^{r}$ value. Data of Fig. 12 displays the frequency variations of the $\alpha$ component. Reported experimental data deal with magnetic fields of up to $56 \mathrm{~T},{ }^{6}$ e.g., with maximum $B / B_{0}$ values of 1.6 . According to the data of Fig. 12, the corresponding frequency shift is $\Delta F=3 \mathrm{~T}$ which is within the reported error bars (since $F_{\alpha}=(944 \pm 4) \mathrm{T}$ for the considered compound). Nevertheless, frequency shift predicted by Eqs. (17), (83) and also recently considered in the case of Bechgaard salts, ${ }^{27}$ could be detected in the future at higher magnetic fields and for orbits involving larger number of reflection events $n_{\eta}^{r}$ such as observed in two-dimensional networks (see Ref. 28).

\section{SUMMARY AND CONCLUSION}

Calculation of transmission and reflection coefficients through a magnetic breakdown junction have been reviewed with the aim of determining the Onsager phase of de Haas-van Alphen oscillations. The problem of the phase divergence of the S-matrix describing wave-function transmission has been addressed by suitable asymptotic analysis. Amplitude of the wave function was then calculated, using approximate and exact models of connected Fermi surfaces, yielding the field-dependent phase offset relevant to de Haas-van Alphen oscillations for Fermi surfaces with magnetic breakdown. As a consequence, experimental de Haas-van Alphen oscillations are not strictly periodic in $B^{-1}$ for orbits with reflections at the magnetic breakdown junctions. Nevertheless, frequency variations, which follow a "universal" field dependence remain small within realistic experimental conditions.

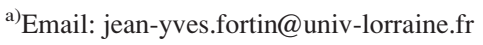

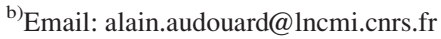

${ }^{1}$ G. P. Mikitik and Y. V. Sharlai, Phys. Rev. Lett. 82, 2147 (1999).

${ }^{2}$ N. J. Fuchs, F. Piéchon, O. M. Goerbig, and G. Montambaux, Eur. Phys. J. B 77, 351 (2010).

${ }^{3}$ A. R. Wright and R. H. McKenzie, Phys. Rev. B 87, 085411 (2013).

${ }^{4}$ J. Y. Fortin and A. Audouard, Eur. Phys. J. B 88, 1 (2015).

${ }^{5}$ A. Slutskin and A. Kadigrobov, Sov. Phys. Solid State 9, 139 (1967).

${ }^{6}$ A. Slutskin, Sov. Phys. - JETP 26, 474 (1968).

${ }^{7}$ W. Huang and P. L. Taylor, Phys. Rev. Lett. 36, 231 (1976).

${ }^{8}$ A. Pippard, Proc. R. Soc. London, Ser. A 270, 1 (1962).

${ }^{9}$ K. Oshima, T. Mori, H. Inokuchi, H. Urayama, H. Yamochi, and G. Saito, Phys. Rev. B 38, 938 (1988).

${ }^{10}$ A. Audouard, J. Y. Fortin, D. Vignolles, R. B. Lyubovskii, E. I. Zhilyaeva, R. N. Lyubovskaya, and E. Canadell, Synth. Met. 171, 51 (2013).

${ }^{11}$ R. Lyubovskii, S. Pesotskii, W. Biberacher, E. Zhilyaeva, A. Bogdanova, and R. Lyubovskaya, Phys. Solid State 50, 1560 (2008).

${ }^{12}$ N. Rosen and C. Zener, Phys. Rev. 40, 502 (1932).

${ }^{13}$ W. Chambers, Phys. Rev. 165, 799 (1968).

${ }^{14}$ B. T. Torosov and N. V. Vitanov, Phys. Rev. A 84, 063411 (2011).

${ }^{15}$ A. Audouard, J. Y. Fortin, D. Vignolles, R. B. Lyubovskii, L. Drigo, F. Duc, G. V. Shilov, G. Ballon, E. I. Zhilyaeva, R. N. Lyubovskaya, and E. Canadell, Europhys. Lett. 97, 57003 (2012).

${ }^{16}$ A. Audouard, J. Y. Fortin, D. Vignolles, R. B. Lyubovskii, L. Drigo, G. V. Shilov, F. Duc, E. I. Zhilyaeva, R. N. Lyubovskaya, and E. Canadell, J. Phys.: Condens. Matter 27, 315601 (2015).

${ }^{17}$ C. S. Lam, J. Math. Phys. 39, 5543 (1998).

${ }^{18}$ A. G. Rojo, see http://arxiv.org/abs/1004.2914v1 for Matrix Exponential Solution of the Landau-Zener Problem Equation.

${ }^{19}$ A. Kholodenko and Z. Silagadze, Phys. Part. Nucl. 43, 882 (2012).

${ }^{20} \mathrm{M}$. H. Holmes, Introduction to Perturbation Methods, Texts in Applied Mathematics (Springer-Verlag, New York, 1995), p. 299.

${ }^{21}$ M. Abramowitz and I. Stegun, Pocketbook of Mathematical Functions (Verlag Harri Deutsch - Thun, Frankfurt am Main, 1984); Asymptotic forms for large argument in the Kummer function are given in 13.5.1. For small h, the two asymptotic expansions for $|y| \simeq r$ and $-r<y<r$ are given, respectively, by 13.5.19 and 13.5.21 with $\cos \theta=y / r$.

${ }^{22}$ A. Kochkin, Sov. Phys. - JETP 27, 324 (1968).

${ }^{23} \mathrm{M}$. Hortaçsu, Heun Functions and Their Uses in Physics (World Scientific Publishing Company, 2012), Chap. 2, pp. 23-39.

${ }^{24}$ M. Kaganov and A. Slutskin, Phys. Rep. 98, 189 (1983), (See Eq. (5.22) and Figs. 17 and 19a).

${ }^{25}$ A. Audouard and J. Y. Fortin, C. R. Phys. 14, 15 (2013).

${ }^{26}$ A. Audouard and J. Y. Fortin, Low Temp. Phys. 40, 344 (2014) [Fiz. Nizk. Temp. 40, 444 (2014) (in Russian)].

${ }^{27}$ G. Montambaux and D. Jerome, C. R. Phys. 17, 376 (2016).

${ }^{28}$ V. N. Laukhin, A. Audouard, J. Y. Fortin, D. Vignolles, T. G. Prokhorova, E. B. Yagubskii, and E. Canadell, Low Temp. Phys. 43, 1 (2017) [Fiz. Nizk. Temp. 43, 33 (2017) (in Russian)].

This article was published in English in the original Russian journal. Reproduced here with stylistic changes by AIP Publishing. 